\title{
Tandem Molybdenum Catalyzed Hydrosilylations: An Expedient Synthesis Of $\beta$-Aryl Aldehydes
}

\author{
Christopher G. Frost,* Benjamin C. Hartley \\ Department of Chemistry, University of Bath, Bath, BA2 7AY, UK.
}

\section{Supporting Information}

General information: IR spectra were recorded on a Perkin-Elmer 1600 FT IR spectrophotometer, using $\mathrm{NaCl}$ discs. ${ }^{1} \mathrm{H}$ NMR spectra were obtained on a Bruker AV250 spectrometer operating at $250 \mathrm{MHz}$, Bruker AV300 spectrometer operating at $300 \mathrm{MHz}$, Bruker AV400 spectrometer operating at $400 \mathrm{MHz}$, unless otherwise noted, with tetramethylsilane as an internal standard. $J$ values are given in $\mathrm{Hz} .{ }^{13} \mathrm{C}$ NMR spectra were obtained on a Bruker Advance 300 spectrometer operating at $75 \mathrm{MHz}$, unless otherwise noted. ${ }^{2} \mathrm{H}$ NMR spectra were obtained on a Bruker Advance 500 spectrometer operating at $77 \mathrm{MHz}$, unless otherwise noted, with an internal standard of $\mathrm{CDCl}_{3}$. All dry solvents were freshly distilled under nitrogen prior to use. Tetrahydrofuran was distilled over alumina column. Petrol refers to petetroleum ether of fractions obtained between 40$60^{\circ} \mathrm{C}$. All other reagents were obtained from commercial suppliers and used as received. All glassware used under anhydrous conditions was dried in an oven and allowed to cool under nitrogen prior to use. All reactions were carried out under argon unless otherwise stated. Flash chromatography was conducted under medium pressure, using matrix 60 silica.

Arylidine and alkylidine compounds were prepared by literature procedures. ${ }^{1,2}$ 


\section{General preparation of 5-Arylidene Meldrum's Acid Derivatives (1a-h):}

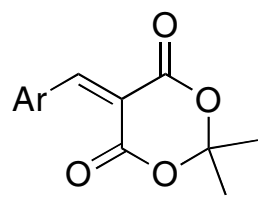

To a solution of Meldrum's acid in water was added aryl aldehyde. The resulting solution was stirred at $75{ }^{\circ} \mathrm{C}$ for 2 hours. After cooling the reaction mixture was filtered via Buckner filtration and washed with water $(100 \mathrm{~mL})$ and petrol $(100 \mathrm{~mL})$. The resulting solid was recrystallised from refluxing ethanol to give the desired compound.

\section{General Preparation of 3-Aryl Propionaldehydes (3a-h):}

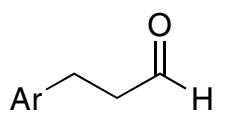

To a solution of Meldrum's acid derivative $(0.5 \mathrm{mmol})$, molybdenum hexacarbonyl (6.6 $\mathrm{mg}, 5 \mathrm{~mol} \%$ ) and $N$-methylmorpholine- $N$-oxide $(5.9 \mathrm{mg}, 10 \mathrm{~mol} \%)$ in tetrahydrofuran (3.0 $\mathrm{mL})$ was added phenylsilane $(185 \mu \mathrm{L}, 1.5 \mathrm{mmol})$. The resulting solution was stirred under an atmosphere of nitrogen at $80{ }^{\circ} \mathrm{C}$ for $16 \mathrm{~h}$. After cooling to room temperature water $(0.5 \mathrm{~mL})$ was added and the solution stirred for 15 minutes. The solution was dissolved in ether $(50 \mathrm{~mL})$ was washed with $1 \mathrm{M} \mathrm{NaOH}(3 \times 50 \mathrm{~mL})$ followed by brine ( 2 x $50 \mathrm{~mL}$ ). The organic phase was extracted, dried over $\mathrm{MgSO}_{4}$ and concentrated in vacuo. The desired product was purified by flash column chromatography. 


\section{5-(4-methoxybenzylidene)-2,2-dimethyl-[1,3]dioxane-4,6-dione (1a): ${ }^{3}$}

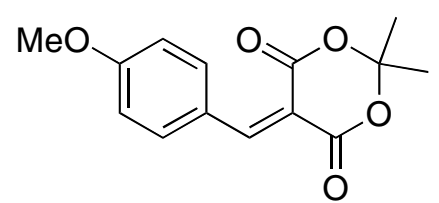

Meldrum's acid (4.0 g, $29.4 \mathrm{mmol}), p$-anisaldehyde (4.7 g, $32.3 \mathrm{mmol})$ in water $(50 \mathrm{~mL})$ was reacted under standard protocol to give the desired compound as a bright yellow crystalline solid (5.58g, 72\% yield); ${ }^{1} \mathrm{H}$ NMR (300 MHz, $\left.\mathrm{CDCl}_{3}\right) \delta 8.34(1 \mathrm{H}, \mathrm{s}, \mathrm{ArCH})$, $8.19(2 \mathrm{H}, \mathrm{d}, J=9.0 \mathrm{~Hz}), 6.95(2 \mathrm{H}, \mathrm{d}, J=9.0 \mathrm{~Hz}), 3.88\left(3 \mathrm{H}, \mathrm{s}, \mathrm{OCH}_{3}\right), 1.76\left(6 \mathrm{H}, \mathrm{s}, \mathrm{CH}_{3}\right)$; ${ }^{13} \mathrm{C}$ NMR $\left(75.5 \mathrm{MHz}, \mathrm{CDCl}_{3}\right) \delta 165.0,164.4,160.8,158.3,138.0,125.1,115.0,111.3$, 104.5, 56.1, 27.9; HRMS (EI) calcd for $\mathrm{C}_{17} \mathrm{H}_{14} \mathrm{O}_{5} \mathrm{Na}[\mathrm{M}+\mathrm{Na}]^{+}: \mathrm{m} / z$ 285.0739, found: $\mathrm{m} / z$ 285.0725 .

\section{5-(4-dimethylaminobenzylidene)-2,2-dimethyl-[1,3]dioxane-4,6-dione (1b): ${ }^{4}$}

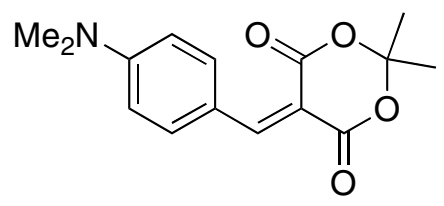

Meldrum's acid (720 mg, $5 \mathrm{mmol})$, 4-dimethylaminobenzaldehyde (821 mg, $5.5 \mathrm{mmol})$ in water $(10 \mathrm{~mL})$ was reacted under standard protocol to give the desired compound as a bright orange solid (1.16 g, 92\% yield); ${ }^{1} \mathrm{H}$ NMR $\left(300 \mathrm{MHz}, \mathrm{CDCl}_{3}\right) \delta 8.31(1 \mathrm{H}, \mathrm{s}$, $\operatorname{ArCH}), 8.25(2 \mathrm{H}, \mathrm{d}, J=9.4 \mathrm{~Hz}, \mathrm{Ar}), 6.70(2 \mathrm{H}, \mathrm{d}, J=9.4 \mathrm{~Hz}, \mathrm{Ar}), 3.15\left(6 \mathrm{H}, \mathrm{s}, \mathrm{N}\left(\mathrm{CH}_{3}\right)_{2}\right)$, $1.76\left(6 \mathrm{H}, \mathrm{s}, \mathrm{CH}_{3}\right) ;{ }^{13} \mathrm{C} \mathrm{NMR}\left(75.5 \mathrm{MHz}, \mathrm{CDCl}_{3}\right) \delta 165.6,161.9,158.4,155.0,139.4$, 120.5, 111.6, 105.4, 103.8, 40.5, 27.7; HRMS (EI) calcd for $\mathrm{C}_{15} \mathrm{H}_{18} \mathrm{NO}_{4}[\mathrm{M}+\mathrm{H}]^{+}: \mathrm{m} / z$ 276.1236, found: $m / z 276.1230$. 
5-(4-(Methylthio)benzylidene)-2,2-dimethyl-1,3-dioxane-4,6-dione (1c):

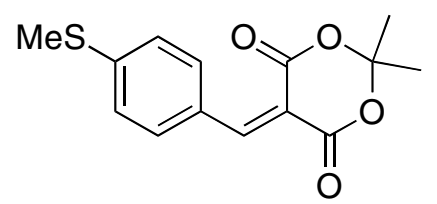

2,2-Dimethyl-[1,3]dioxane-4,6-dione $\quad(3.41 \quad \mathrm{~g}, \quad 23.65 \quad \mathrm{mmol}) \quad$ and 4(methylthio)benzaldehyde $(3.00 \mathrm{~g}, 19.71 \mathrm{mmol})$, were reacted under the standard protocol to generate the title compound $(4.11 \mathrm{~g}, 75 \%$ yield) as a yellow solid; $\mathrm{mp}(\mathrm{EtOH})$ 126-128 ${ }^{\circ} \mathrm{C} ;{ }^{1} \mathrm{H}$ NMR $\left(300 \mathrm{MHz}, \mathrm{CDCl}_{3}\right) \delta 8.39(1 \mathrm{H}, \mathrm{s}, \mathrm{CH}) ; 8.11(2 \mathrm{H}, \mathrm{d}, J 6.78 \mathrm{~Hz}, \mathrm{CH}$ Ar); $7.32(2 \mathrm{H}, \mathrm{d}, J 6.78 \mathrm{~Hz}, \mathrm{CH} \mathrm{Ar}) ; 2.58\left(3 \mathrm{H}, \mathrm{s}, \mathrm{SCH}_{3}\right), 1.83\left(6 \mathrm{H}, \mathrm{s}, \mathrm{CH}_{3}\right) ;{ }^{13} \mathrm{C} \mathrm{NMR}$ $\left(75.5 \mathrm{MHz}, \mathrm{CDCl}_{3}\right) \delta 163.6,160.1,157.4,148.4,134.6,127.7,124.7,112.4,104.2,27.5$, 14.4; IR $\left(\mathrm{KBr}, \mathrm{cm}^{-1}\right)$ v 3099, $2986\left(\mathrm{CO}-\mathrm{CH}_{3}\right) ; 1750,1711(\mathrm{C}=\mathrm{O}) ; 1577\left(\mathrm{~S}-\mathrm{CH}_{3}\right)$.

\section{5-(4-nitrobenzylidene)-2,2-dimethyl-[1,3]dioxane-4,6-dione (1d): ${ }^{5}$}

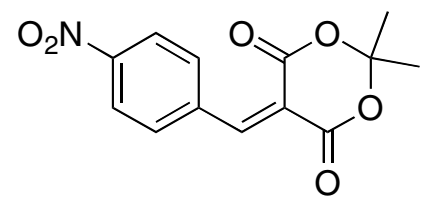

Meldrum's acid (4 g, $29.4 \mathrm{mmol})$, 4-nitrobenzaldehyde (4.88 g, $32.3 \mathrm{mmol})$ and water $(50 \mathrm{~mL})$ was reacted under standard protocol to give the desired product as a pale orange solid (6.78 g, $83 \%)$; ${ }^{1} \mathrm{H}$ NMR (300 MHz, $\left.\mathrm{CDCl}_{3}\right) \delta 8.45(1 \mathrm{H}, \mathrm{s}, \mathrm{ArCH}), 8.30(2 \mathrm{H}, \mathrm{d}, J=$ $8.7 \mathrm{~Hz}, \mathrm{Ar}), 8.06(2 \mathrm{H}, \mathrm{d}, J=8.7 \mathrm{~Hz}, \mathrm{Ar}), 1.84\left(6 \mathrm{H}, \mathrm{s}, \mathrm{CH}_{3}\right) ;{ }^{13} \mathrm{C} \mathrm{NMR}(75.5 \mathrm{MHz}$, $\left.\mathrm{CDCl}_{3}\right) \delta 162.4,154.8,137.8,133.5,124.0,118.9,105.7,28.24$. 


\section{5-(benzo-[1,3]dioxol-4-ylmethylene)-2,2-dimethyl-[1,3]dioxane-4,6-dione (1e):}

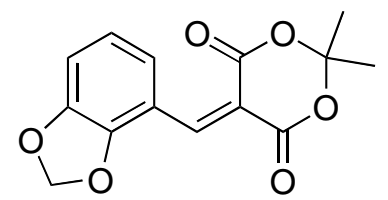

Meldrum's acid (4.0 g, 29.4 mmol), 2,3-(methylenedioxy)benzaldehyde (4.8 g, 32.3 mmol) in water $(50 \mathrm{~mL})$ was reacted under standard protocol to give the desired compound as a bright yellow crystalline solid $\left(7.04 \mathrm{~g}, 73 \%\right.$ yield); $v_{\max }$ (nujol) $/ \mathrm{cm}^{-1}$; 1755, $1725(\mathrm{C}=\mathrm{O}), 1276,1256(\mathrm{O}-\mathrm{C}-\mathrm{O}) ;{ }^{1} \mathrm{H}$ NMR $\left(300 \mathrm{MHz}, \mathrm{CDCl}_{3}\right) \delta 8.43(1 \mathrm{H}, \mathrm{s}$, $\operatorname{ArCH}), 7.50\left(1 \mathrm{H}, \mathrm{dd}, J^{1}=7.5 \mathrm{~Hz}, J^{2}=1.5 \mathrm{~Hz}, \mathrm{Ar}\right), 6.95\left(1 \mathrm{H}, \mathrm{dd}, J^{l}=7.5 \mathrm{~Hz}, J^{2}=1.5\right.$ $\mathrm{Hz}, \mathrm{Ar}), 6.88(1 \mathrm{H}, \mathrm{t}, J=7.5 \mathrm{~Hz}, \mathrm{Ar}), 6.06\left(2 \mathrm{H}, \mathrm{s}, \mathrm{OCH}_{2} \mathrm{O}\right), 1.80\left(6 \mathrm{H}, \mathrm{s}, \mathrm{CH}_{3}\right) ;{ }^{13} \mathrm{C} \mathrm{NMR}$ $\left(75.5 \mathrm{MHz}, \mathrm{CDCl}_{3}\right) \delta 163.2,160.0,149.8,149.6,148.2,124.1,122.1,166.4,115.4$, 113.1, 105.1, 102.1, 28.0; HRMS (EI) calcd for $\mathrm{C}_{14} \mathrm{H}_{12} \mathrm{O}_{6} \mathrm{Na}[\mathrm{M}+\mathrm{Na}]^{+}: \mathrm{m} / z$ 299.0532, found: $m / z 299.0530$.

\section{5-(2,5-dimethoxybenzylidene)-2,2-dimethyl-[1,3]dioxane-4,6-dione (1f): ${ }^{6}$}<smiles>COc1ccc(OC)c(C=C2C(=O)OC(C)(C)OC2=O)c1</smiles>

Meldrum's acid (1.44 g, $10 \mathrm{mmol}), 2,5$-dimethoxybenzaldehyde $(1.83 \mathrm{~g}, 11 \mathrm{mmol})$ and water $(20 \mathrm{~mL})$ was reacted under standard protocol to give the desired compound as an orange solid (2.15 g, $73 \%) ;{ }^{1} \mathrm{H}$ NMR $\left(300 \mathrm{MHz}, \mathrm{CDCl}_{3}\right) \delta 8.72(1 \mathrm{H}, \mathrm{s}, \mathrm{ArCH}), 7.67(1 \mathrm{H}$, d, $J=3.0 \mathrm{~Hz}, \mathrm{Ar}), 7.08\left(1 \mathrm{H}, \mathrm{dd}, J^{l}=9.0 \mathrm{~Hz}, J^{2}=3.0 \mathrm{~Hz}, \mathrm{Ar}\right), 6.90(1 \mathrm{H}, \mathrm{d}, J=9.0 \mathrm{~Hz}$, $\mathrm{Ar}), 3.85\left(3 \mathrm{H}, \mathrm{s}, \mathrm{OCH}_{3}\right), 3.79\left(3 \mathrm{H}, \mathrm{s}, \mathrm{OCH}_{3}\right), 1.80\left(6 \mathrm{H}, \mathrm{s}, \mathrm{CH}_{3}\right) ;{ }^{13} \mathrm{C} \mathrm{NMR}(75.5 \mathrm{MHz}$, $\left.\mathrm{CDCl}_{3}\right) \delta 163.7,160.5,154.8,153.3,152.8,122.5,121.9,116.5,115.4,112.4,104.8$, 
56.5, 56.3, 27.9; HRMS (EI) calcd for $\mathrm{C}_{15} \mathrm{H}_{16} \mathrm{O}_{6} \mathrm{Na}[\mathrm{M}+\mathrm{Na}]^{+}: \mathrm{m} / z$ 315.0845, found: $\mathrm{m} / \mathrm{z}$ 315.0856 .

\section{5-(benzo-[1,3]dioxol-5-ylmethylene)-2,2-dimethyl-[1,3]dioxane-4,6-dione (1g): ${ }^{7}$}

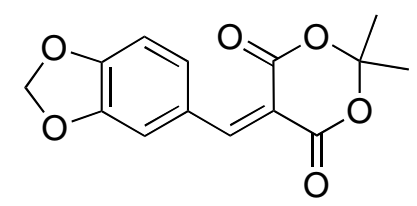

Meldrum's acid $(4.0 \mathrm{~g}, 29.4 \mathrm{mmol})$, piperonal $(4.8 \mathrm{~g}, 32.3 \mathrm{mmol})$ in water $(50 \mathrm{~mL})$ was reacted under standard protocol to give the desired compound as a bright yellow crystalline solid (7.94 g, $82 \%$ yield); ${ }^{1} \mathrm{H}$ NMR $\left(300 \mathrm{MHz}, \mathrm{CDCl}_{3}\right) \delta 8.31(1 \mathrm{H}, \mathrm{s}, \mathrm{ArCH})$, $8.06(1 \mathrm{H}, \mathrm{d}, J=1.9 \mathrm{~Hz}, \mathrm{Ar}), 7.54\left(1 \mathrm{H}, \mathrm{dd}, J^{l}=8.3 \mathrm{~Hz}, J^{2}=1.9 \mathrm{~Hz}, \mathrm{Ar}\right), 6.90(1 \mathrm{H}, \mathrm{d}, J=$ $8.3 \mathrm{~Hz}, \mathrm{Ar}), 6.09\left(2 \mathrm{H}, \mathrm{s}, \mathrm{OCH}_{2} \mathrm{O}\right), 1.78\left(6 \mathrm{H}, \mathrm{s}, \mathrm{CH}_{3}\right) ;{ }^{13} \mathrm{C} \mathrm{NMR}\left(75.5 \mathrm{MHz}, \mathrm{CDCl}_{3}\right) \delta$ 164.3, 160.7, 158.3, 153.6, 148.7, 134.6, 126.8, 112.8, 111.7, 108.9, 104.7, 102.8, 27.9; HRMS (EI) calcd for $\mathrm{C}_{14} \mathrm{H}_{12} \mathrm{O}_{6} \mathrm{Na}[\mathrm{M}+\mathrm{Na}]^{+}: \mathrm{m} / z$ 299.0532, found: $\mathrm{m} / \mathrm{z} 299.0534$.

\section{5-(2,3,4-trimethoxybenzylidene)-2,2-dimethyl-[1,3]dioxane-4,6-dione (1h): ${ }^{8}$}<smiles>COc1ccc(C=C2C(=O)OC(C)(C)OC2=O)c(OC)c1OC</smiles>

Meldrum's acid (721 mg, $5 \mathrm{mmol}), 2,3,4$-trimethoxybenzaldehyde (1.08 g, $5.5 \mathrm{mmol})$ and water $(10 \mathrm{~mL})$ was reacted under standard protocol to give the desired compound as a yellow solid (1.11 g, $69 \%) ;{ }^{1} \mathrm{H}$ NMR $\left(300 \mathrm{MHz}, \mathrm{CDCl}_{3}\right) \delta 8.72(1 \mathrm{H}, \mathrm{s}, \mathrm{ArCH}), 8.16$ $(1 \mathrm{H}, \mathrm{d}, J=9.0 \mathrm{~Hz}, \mathrm{Ar}), 6.72(1 \mathrm{H}, \mathrm{d}, J=9.0 \mathrm{~Hz}, \mathrm{Ar}), 3.99\left(3 \mathrm{H}, \mathrm{s}, \mathrm{OCH}_{3}\right), 3.94(3 \mathrm{H}, \mathrm{s}$, $\left.\mathrm{OCH}_{3}\right), 3.84\left(3 \mathrm{H}, \mathrm{s}, \mathrm{OCH}_{3}\right), 1.78\left(6 \mathrm{H}, \mathrm{s}, \mathrm{CH}_{3}\right) ;{ }^{13} \mathrm{C} \mathrm{NMR}\left(75.5 \mathrm{MHz}, \mathrm{CDCl}_{3}\right) \delta$ 164.1, 161.0, 159.6, 156.4, 153.0, 141.8, 129.6, 119.4, 112.6, 107.3, 104.6, 62.5, 61.4, 56.7, 27.9; HRMS (EI) calcd for $\mathrm{C}_{16} \mathrm{H}_{18} \mathrm{O}_{7} \mathrm{Na}[\mathrm{M}+\mathrm{Na}]^{+}: \mathrm{m} / z$ 345.0950, found: $m / z 345.0931$. 


\section{2,2-dimethyl-5-(4-nitrobenzyl)-1,3-dioxane-4,6-dione (2d):}

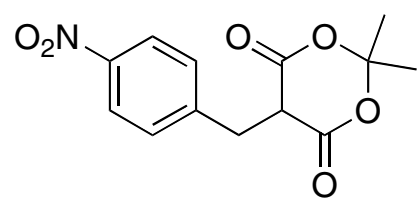

To a solution of 5-(4-nitrobenzylidene)-2,2-dimethyl-[1,3]dioxane-4,6-dione (1 g, 3.6 mmol) in dichlomethane $(25 \mathrm{~mL})$ was added glacial acetic acid $(4 \mathrm{~mL})$. The resulting solution was cooled to $0^{\circ} \mathrm{C}$ and sodium borohydride $(0.41 \mathrm{~g}, 10.8 \mathrm{mmol})$ was added in portions. The resulting solution was allowed to reach ambient temperature and stirred for 1 hour. The solution was dissolved in dichloromethane $(25 \mathrm{~mL})$ and washed with three times with brine $(50 \mathrm{~mL})$ and twice with water $(50 \mathrm{~mL})$. The organic phase was extracted, dried over $\mathrm{MgSO}_{4}$ and concentrated in vacuo to give the desired product without further purification as a pale yellow solid $(881 \mathrm{mg}, 87 \%) ; v_{\max }($ nujol $) / \mathrm{cm}^{-1}$; 1779, $1742(\mathrm{C}=\mathrm{O}) ; 1348(\mathrm{~N}-\mathrm{O}) ;{ }^{1} \mathrm{H}$ NMR $\left(300 \mathrm{MHz}, \mathrm{CDCl}_{3}\right) \delta 8.13(2 \mathrm{H}, \mathrm{d}, J=8.7 \mathrm{~Hz}$, Ar), $7.53(2 \mathrm{H}, \mathrm{d}, J=8.7, \mathrm{Ar}), 3.83(1 \mathrm{H}, \mathrm{t}, J=5.3, \mathrm{CH}), 3.57\left(2 \mathrm{H}, \mathrm{d}, J=5.3 \mathrm{~Hz}, \mathrm{ArCH}_{2}\right)$, $1.79\left(3 \mathrm{H}, \mathrm{s}, \mathrm{CH}_{3}\right), 1.67\left(3 \mathrm{H}, \mathrm{s}, \mathrm{CH}_{3}\right) ;{ }^{13} \mathrm{C} \mathrm{NMR}\left(75.5 \mathrm{MHz}, \mathrm{CDCl}_{3}\right) \delta 165.0,147.5$, 145.1, 131.3, 124.1, 105.8, 48.1, 31.8, 28.8, 27.4; HRMS (EI) calcd for $\mathrm{C}_{13} \mathrm{H}_{12} \mathrm{NO}_{6}$ [M$\mathrm{H}]^{-}: m / z$ 278.0665, found: $m / z 278.0674$.

\section{3-(4-methoxyphenyl)-propanal (3a):}

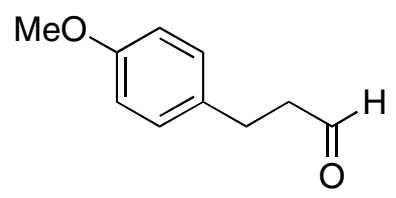

5-(4-methoxybenzylidene)-2,2-dimethyl-[1,3]dioxane-4,6-dione (131.0 mg, $0.5 \mathrm{mmol}$ ) was reacted under standard protocol to give the desired product, purified by flash column chromatography (petrol : ethyl acetate 11:1), as a colourless oil (70.1 mg, 85\% yield); ${ }^{1} \mathrm{H}$ NMR (300 MHz, $\left.\mathrm{CDCl}_{3}\right) \delta 9.80(1 \mathrm{H}, \mathrm{t}, J=1.5 \mathrm{~Hz}, \mathrm{CHO}), 7.11(2 \mathrm{H}, \mathrm{d}, J=8.7 \mathrm{~Hz}, \mathrm{Ar})$, 
$6.84(2 \mathrm{H}, \mathrm{d}, J=8.7 \mathrm{~Hz}, \mathrm{Ar}), 3.78\left(3 \mathrm{H}, \mathrm{s}, \mathrm{CH}_{3}\right), 2.91\left(2 \mathrm{H}, \mathrm{t}, J=7.2 \mathrm{~Hz}, \mathrm{ArCH}_{2}\right), 2.77-$ $2.72\left(2 \mathrm{H}, \mathrm{m}, \mathrm{CH}_{2}\right) ;{ }^{13} \mathrm{C}$ NMR $\left(75.5 \mathrm{MHz}, \mathrm{CDCl}_{3}\right) \delta 202.3,158.5,132.7,129.7,128.4$, 60.8, 55.7, 46.0, 27.7, 21.5, 14.6; HRMS (EI) calcd for $\mathrm{C}_{10} \mathrm{H}_{12} \mathrm{O}_{2} \mathrm{Na}[\mathrm{M}+\mathrm{Na}]^{+}: \mathrm{m} / \mathrm{z}$ 187.0735, found : $\mathrm{m} / \mathrm{z} 187.0738$.

\section{3-(4-dimethylaminobenzene)-propanal (3b):}

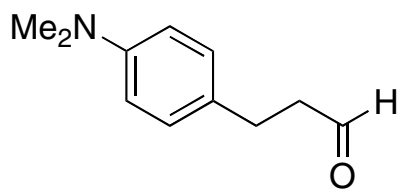

5-(4-dimethylaminobenzylidene)-2,2-dimethyl-[1,3]dioxane-4,6-dione (125.6, $0.5 \mathrm{mmol}$ ) was reacted under standard protocol to give the desired product, purified by flash column chromatography (petrol : ethyl acetate (11:1), as a colourless oil (71.8 mg, 81\% yield); $v_{\max }$ (neat) $/ \mathrm{cm}^{-1} ; 2729(\mathrm{C}-\mathrm{H}) ; 1722(\mathrm{C}=\mathrm{O}) ; 1164,1135(\mathrm{~N}-\mathrm{C}) ;{ }^{1} \mathrm{H}$ NMR $\left(250 \mathrm{MHz}, \mathrm{CDCl}_{3}\right) \delta 9.82(1 \mathrm{H}, \mathrm{t}, J=1.5 \mathrm{~Hz}, \mathrm{CHO}), 7.07(2 \mathrm{H}, \mathrm{d}, J=8.7 \mathrm{~Hz}, \mathrm{Ar})$, $6.70(2 \mathrm{H}, \mathrm{d}, J=8.7 \mathrm{~Hz}, \mathrm{Ar}), 2.92-2.86(8 \mathrm{H}, \mathrm{m}), 2.76-2.70\left(2 \mathrm{H}, \mathrm{m}, \mathrm{CH}_{2}\right) ;{ }^{13} \mathrm{C}$ NMR $(75.5$ $\mathrm{MHz}, \mathrm{CDCl}_{3}$ ) $\delta 202.7,149.7,129.3,128.6,113.4,46.1,41.2,27.6$; HRMS (EI) calcd for $\mathrm{C} 11 \mathrm{H} 16 \mathrm{NO}[\mathrm{M}+\mathrm{H}]^{+}: \mathrm{m} / \mathrm{z}$ 178.1232, found $: m / z$ 178.1228.

\subsection{6: 3-(4-thiomethylbenzene)-propanal (3c):}

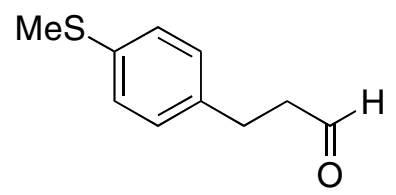

5-(4-thiomethylbenzylidene)-2,2-dimethyl-[1,3]dioxane-4,6-dione (139 mg, $0.5 \mathrm{mmol})$ was reacted under standard conditions to give the desired product, purified by flash column chromatography (petrol : ethyl acetate 11:1), as a colourless oil (44 mg, 66\%); $v_{\max }($ neat $) / \mathrm{cm}^{-1} ; 2727(\mathrm{C}-\mathrm{H}) ; 1722(\mathrm{C}=\mathrm{O}) ; 1583(\mathrm{C}-\mathrm{S}) ;{ }^{1} \mathrm{H}$ NMR $\left(300 \mathrm{MHz}, \mathrm{CDCl}_{3}\right) \delta$ 
$9.81(1 \mathrm{H}, \mathrm{t}, J=1.5 \mathrm{~Hz}, \mathrm{CHO}), 7.20(2 \mathrm{H}, \mathrm{d}, J=8.7 \mathrm{~Hz}, \mathrm{Ar}), 7.12(2 \mathrm{H}, \mathrm{d}, J=8.7 \mathrm{~Hz}, \mathrm{Ar})$, $2.92\left(2 \mathrm{H}, \mathrm{t}, J=7.2 \mathrm{~Hz}, \mathrm{ArCH}_{2}\right), 2.79-2.73\left(2 \mathrm{H}, \mathrm{m}, \mathrm{CH}_{2}\right) ;{ }^{13} \mathrm{C} \mathrm{NMR}\left(75.5 \mathrm{MHz}, \mathrm{CDCl}_{3}\right) \delta$ 201.9, 137.7, 136.5, 129.3, 127.5, 45.7, 31.4, 28.0, 16.6, HRMS (EI) calcd for $\mathrm{C}_{10} \mathrm{H}_{16} \mathrm{NOS}\left[\mathrm{M}+\mathrm{NH}_{4}\right]^{+}: \mathrm{m} / \mathrm{z}$ 198.0947, found : $\mathrm{m} / \mathrm{z} 198.948$.

\section{3-(4-nitrophenyl)-propanal (3d): ${ }^{10}$}

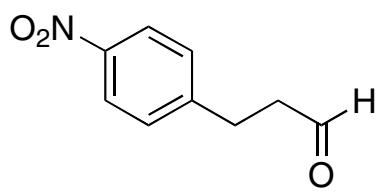

5-(4-nitrobenzylidene)-2,2-dimethyl-[1,3]dioxane-4,6-dione (138.6 mg, $0.5 \mathrm{mmol}$ ) was reacted under standard conditions to give the desired product, purified by flash column chromatography (petrol : ethyl acetate 6:1), as colourless crystals (32.8 mg, $37 \%$ ); 5-(4nitrobenzyl)-2,2-dimethyl-[1,3]dioxane-4,6-dione $(139.6 \mathrm{mg}, 0.5 \mathrm{mmol})$ was reacted under standard conditions to give the desired product, purified by flash column chromatography (petrol : ethyl acetate 6:1), as colourless crystals (81.6 mg, $91 \%) ;{ }^{1} \mathrm{H}$ NMR (300 MHz, CDCl $) \delta 9.82(1 \mathrm{H}, \mathrm{t}, J=1.1 \mathrm{~Hz}, \mathrm{CHO}), 8.14(2 \mathrm{H}, \mathrm{d}, J=8.7 \mathrm{~Hz}, \mathrm{Ar})$, $7.36(2 \mathrm{H}, \mathrm{d}, J=8.7 \mathrm{~Hz}, \mathrm{Ar}), 3.05\left(2 \mathrm{H}, \mathrm{t}, 7.2 \mathrm{~Hz}, \mathrm{ArCH}_{2}\right), 2.88-2.83\left(2 \mathrm{H}, \mathrm{m}, \mathrm{CH}_{2}\right) ;{ }^{13} \mathrm{C}$ NMR (75.5 MHz, $\left.\mathrm{CDCl}_{3}\right) \delta$ 200.6, 148.7, 147.0, 129.7, 124.2, 44.9, 28.2; HRMS (EI) calcd for $\mathrm{C}_{9} \mathrm{H}_{10} \mathrm{NO}_{3}[\mathrm{M}+\mathrm{H}]^{+}: \mathrm{m} / \mathrm{z}$ 180.0661, found : $\mathrm{m} / \mathrm{z} 180.0660$.

\section{3-(benzo[1,3]-dioxol-4-yl)-propanal (3e):}

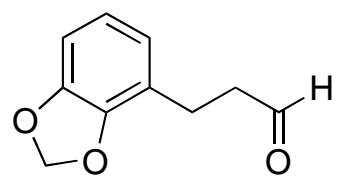

5-(benzo[1,3]dioxol-4-ylmethylene)-2,2-dimethyl-1,3-dioxane-4,6-dione (138.1 mg, 0.5 mmol) was reacted under standard conditions to give the desired product, purified by 
flash column chromatography (petrol : ethyl acetate (11:1), as a colourless oil $(69.4 \mathrm{mg}$, $78 \%) ; v_{\max }($ neat $) / \mathrm{cm}^{-1} ; 2729(\mathrm{C}-\mathrm{H}) ; 1724(\mathrm{C}=\mathrm{O}) ; 1252(\mathrm{C}-\mathrm{O}) ;{ }^{1} \mathrm{H}$ NMR $(300 \mathrm{MHz}$, $\left.\mathrm{CDCl}_{3}\right) \delta 9.82(1 \mathrm{H}, \mathrm{t}, J=1.5 \mathrm{~Hz}, \mathrm{CHO}), 6.79-6.65(3 \mathrm{H}, \mathrm{m}, \mathrm{Ar}), 5.94\left(2 \mathrm{H}, \mathrm{s},-\mathrm{OCH}_{2} \mathrm{O}-\right)$,

2.95-2.89 (2H, m, $\left.\mathrm{ArCH}_{2}\right), 2.82-2.76\left(2 \mathrm{H}, \mathrm{m}, \mathrm{CH}_{2}\right) ;{ }^{13} \mathrm{C} \mathrm{NMR}\left(75.5 \mathrm{MHz}, \mathrm{CDCl}_{3}\right) \delta$ 202.0, 147.6, 145.8, 128.2, 122.8, 122.1, 107.4, 101.0, 43.7, 22.7; HRMS (EI) calcd for $\mathrm{C}_{10} \mathrm{H}_{10} \mathrm{O}_{3} \mathrm{Na}[\mathrm{M}+\mathrm{Na}]^{+}: \mathrm{m} / \mathrm{z} 201.0528$, found : $\mathrm{m} / \mathrm{z} 201.0524$.

\section{3-(2,5-dimethoxyphenyl)-propanal (3f):}

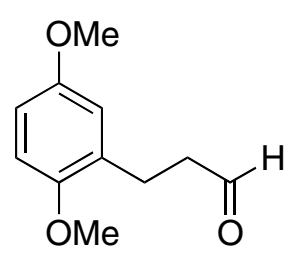

5-(2,5-dimethoxybenzylidene)-2,2-dimethyl-1,3-dioxane-4,6-dione (146.1 mg, $0.5 \mathrm{mmol}$ ) was reacted under standard protocol to give the desired product, purified by flash column chromatography (petrol : ethyl acetate 11:1), as a colourless oil (73.4 mg, $76 \%$ ); $v_{\max }$ (neat) $/ \mathrm{cm}^{-1} ; 2729(\mathrm{C}-\mathrm{H}) ; 1723(\mathrm{C}=\mathrm{O}) ; 1225(\mathrm{C}-\mathrm{O}) ;{ }^{1} \mathrm{H}$ NMR (300 MHz, $\left.\mathrm{CDCl}_{3}\right) \delta 9.80$ $(1 \mathrm{H}, \mathrm{t}, J=1.5 \mathrm{~Hz}, \mathrm{CHO}), 6.78-6.69(3 \mathrm{H}, \mathrm{m}, \mathrm{Ar}), 3.77\left(3 \mathrm{H}, \mathrm{s}, \mathrm{OCH}_{3}\right), 3.75\left(3 \mathrm{H}, \mathrm{s}, \mathrm{OCH}_{3}\right)$, $2.92\left(2 \mathrm{H}, \mathrm{t}, J=7.2 \mathrm{~Hz}, \mathrm{ArCH}_{2}\right), 2.74-2.68\left(2 \mathrm{H}, \mathrm{m}, \mathrm{CH}_{2}\right) ;{ }^{13} \mathrm{C} \mathrm{NMR}\left(75.5 \mathrm{MHz}, \mathrm{CDCl}_{3}\right) \delta$ 202.7, 153.9, 152.0, 130.2, 116.8, 111.9, 111.5, 56.1, 44.3, 24.1; HRMS (EI) calcd for $\mathrm{C}_{11} \mathrm{H}_{14} \mathrm{O}_{3} \mathrm{Na}[\mathrm{M}+\mathrm{Na}]^{+}: \mathrm{m} / z, 217.0841$, found : $\mathrm{m} / \mathrm{z}, 217.0840$.

\section{3-(benzo[1,3]dioxol-5-yl)propanal (3g): ${ }^{11}$}<smiles>O=CCCc1ccc2c(c1)OCO2</smiles> 
5-(benzo[1,3]dioxol-5-ylmethyl)-2,2-dimethyl-1,3-dioxane-4,6-dione (138.1 mg, 0.5 mmol) was reacted under standard protocol to give the desired product, purified by column chromatography (petrol : ethyl acetate 6:1), as a colourless oil (58.3 mg, $65 \%$ ); ${ }^{1} \mathrm{H}$ NMR $\left(400 \mathrm{MHz}, \mathrm{CDCl}_{3}\right) \delta 9.79(1 \mathrm{H}, \mathrm{t}, J=1.5 \mathrm{~Hz}, \mathrm{CHO}), 6.72(1 \mathrm{H}, \mathrm{d}, J=7.8 \mathrm{~Hz}$, $\mathrm{Ar})$, 6.68-6.62 (2H, m, Ar), $5.91\left(2 \mathrm{H}, \mathrm{s}, \mathrm{OCH}_{2} \mathrm{O}\right), 2.87\left(2 \mathrm{H}, \mathrm{t}, J=7.3 \mathrm{~Hz}, \mathrm{ArCH}_{2}\right), 2.74-$ $2.70\left(2 \mathrm{H}, \mathrm{m}, \mathrm{CH}_{2}\right) ;{ }^{13} \mathrm{C} \mathrm{NMR}\left(75.5 \mathrm{MHz}, \mathrm{CDCl}_{3}\right) \delta 202.2,148.1,146.4,134.5,121.5$, 109.2, 108.7, 101.3, 46.0, 28.3; HRMS (EI) calcd for $\mathrm{C}_{10} \mathrm{H}_{10} \mathrm{O}_{3} \mathrm{Na}[\mathrm{M}+\mathrm{Na}]^{+}: \mathrm{m} / z$ 201.0528, found : $m / z 201.0528$.

\section{3-(2,3,4-trimethoxyphenyl)-propanal (3h):}

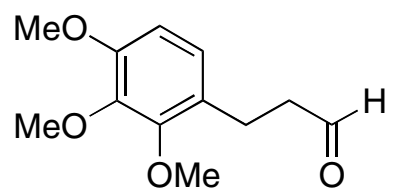

5-(2,3,4-trimethoxybenzylidene)-2,2-dimethyl-1,3-dioxane-4,6-dione (161.2 mg, 0.5 mmol) was reacted under standard protocol to give the desired product, purified by column chromatography (petrol : ethyl acetate (11:1), as a colourless oil (53.3 mg, $48 \%$ ); $v_{\max }($ neat $) / \mathrm{cm}^{-1} ; 2728(\mathrm{C}-\mathrm{H}) ; 1723(\mathrm{C}=\mathrm{O}) ; 1248(\mathrm{O}-\mathrm{C}) ;{ }^{1} \mathrm{H}$ NMR $\left(300 \mathrm{MHz}, \mathrm{CDCl}_{3}\right) \delta$ $9.78(1 \mathrm{H}, \mathrm{t}, J=1.5 \mathrm{~Hz}, \mathrm{CHO}), 6.80(1 \mathrm{H}, \mathrm{d}, J=8.7 \mathrm{~Hz}, \mathrm{Ar}), 6.57(1 \mathrm{H}, \mathrm{d}, J=8.7 \mathrm{~Hz}, \mathrm{Ar})$, $3.87\left(3 \mathrm{H}, \mathrm{s}, \mathrm{OCH}_{3}\right), 3.84\left(3 \mathrm{H}, \mathrm{s}, \mathrm{OCH}_{3}\right), 3.81\left(3 \mathrm{H}, \mathrm{s}, \mathrm{OCH}_{3}\right), 2.86(2 \mathrm{H}, \mathrm{t}, J=7.5 \mathrm{~Hz}$, $\left.\mathrm{ArCH}_{2}\right), 2.72-2.66\left(2 \mathrm{H}, \mathrm{m}, \mathrm{CH}_{2}\right) ;{ }^{13} \mathrm{C} \mathrm{NMR}\left(75.5 \mathrm{MHz}, \mathrm{CDCl}_{3}\right) \delta 202.7,152.9,152.2$, 142.6, 126.6, 124.2, 107.5, 61.2, 61.1, 56.4, 45.1, 23.2; HRMS (EI) calcd for $\mathrm{C}_{12} \mathrm{H}_{16} \mathrm{O}_{4} \mathrm{Na}$ $[\mathrm{M}+\mathrm{Na}]^{+}: m / z$ 247.0946, found : $m / z 247.0937$. 


\section{Preparation of $\alpha, \alpha$-dideuterated-3-(4-methoxyphenyl) propionaldehyde (4a):}

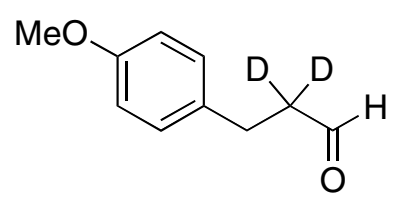

To a solution of 3-(4-methoxyphenyl)-propanal (3a) (0.5 mmol), molybdenum hexacarbonyl (6.6 mg, $5 \mathrm{~mol} \%)$ and $N$-methylmorpholine- $N$-oxide $(5.9 \mathrm{mg}, 10 \mathrm{~mol} \%)$ in tetrahydrofuran $(3.0 \mathrm{~mL})$ was added phenylsilane $(185 \mu \mathrm{L}, 1.5 \mathrm{mmol})$. The resulting solution was stirred under an atmosphere of nitrogen at $80{ }^{\circ} \mathrm{C}$ for $16 \mathrm{~h}$. After cooling to room temperature $\mathrm{D}_{2} \mathrm{O}(0.5 \mathrm{~mL})$ was added and the solution stirred for 15 minutes. The solution was dissolved in ether $(50 \mathrm{~mL})$ was washed with $1 \mathrm{M} \mathrm{NaOH}(3 \times 50 \mathrm{~mL})$ followed by brine $\left(2 \times 50 \mathrm{~mL}\right.$ ). The organic phase was extracted, dried over $\mathrm{MgSO}_{4}$ and concentrated in vacuo. The desired product was isolated by flash column chromatography (petrol : ethyl acetate 11:1) as a colourless oil $(61.5 \mathrm{mg}, 75 \%) ; 85 \%$ deuterium

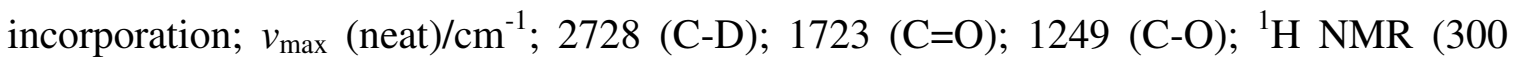
$\left.\mathrm{MHz}, \mathrm{CDCl}_{3}\right) \delta 9.82(1 \mathrm{H}, \mathrm{s}, \mathrm{CHO}), 7.11(2 \mathrm{H}, \mathrm{d}, J=9.04 \mathrm{~Hz}, \mathrm{Ar}), 6.83(2 \mathrm{H}, \mathrm{d}, J=9.04$ $\mathrm{Hz}, \mathrm{Ar}), 3.79\left(3 \mathrm{H}, \mathrm{s}, \mathrm{CH}_{3}\right), 2.89\left(2 \mathrm{H}, \mathrm{s}, \mathrm{CH}_{2}\right) ;{ }^{2} \mathrm{H} \mathrm{NMR}(77 \mathrm{MHz}, \mathrm{DCM}) \delta 2.62(\mathrm{~s}) ;{ }^{13} \mathrm{C}$ NMR (75.5 MHz, $\left.\mathrm{CDCl}_{3}\right) \delta 202.5,158.5,132.7,129.6,114.4,55.7,27.6$; HRMS (EI) calcd for $\mathrm{C}_{10} \mathrm{H}_{10} \mathrm{D}_{2} \mathrm{ONa}[\mathrm{M}+\mathrm{Na}]^{+}: \mathrm{m} / z, 189.0855$, found: $m / z, 189.0855$. 


\section{1,3-dideuterated-3-(4-methoxyphenyl) propionaldehyde (5a):}

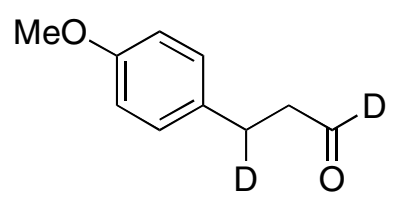

To a solution of 3-(4-methoxyphenyl)-propanal (3a) (0.5 mmol), molybdenum hexacarbonyl (6.6 mg, $5 \mathrm{~mol} \%$ ) and $N$-methylmorpholine- $N$-oxide $(5.9 \mathrm{mg}, 10 \mathrm{~mol} \%$ ) in tetrahydrofuran $(3.0 \mathrm{~mL})$ was added trideuteriophenylsilane $(185 \mu \mathrm{L}, 1.5 \mathrm{mmol})$. The resulting solution was stirred under an atmosphere of nitrogen at $80{ }^{\circ} \mathrm{C}$ for $16 \mathrm{~h}$. After cooling to room temperature water $(0.5 \mathrm{~mL})$ was added and the solution stirred for 15 minutes. The solution was dissolved in ether $(50 \mathrm{~mL})$ was washed with $1 \mathrm{M} \mathrm{NaOH}(3 \mathrm{x}$ $50 \mathrm{~mL})$ followed by brine $(2 \times 50 \mathrm{~mL})$. The organic phase was extracted, dried over $\mathrm{MgSO}_{4}$ and concentrated in vacuo. The desired product was isolated by flash column chromatography (petrol : ethyl acetate 11:1) as a colourless oil (65.1 mg, $79 \%) ; 100 \%$

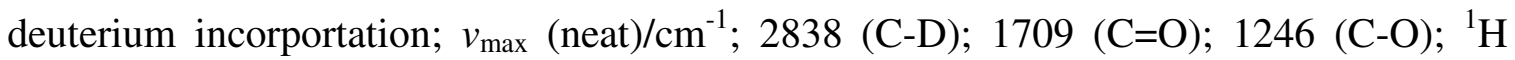
NMR (300 MHz, CDCl $) \delta 7.11(2 \mathrm{H}, \mathrm{d}, J=9.04 \mathrm{~Hz}, \mathrm{Ar}), 6.84(2 \mathrm{H}, \mathrm{d}, J=8.67 \mathrm{~Hz}), 3.79$ $\left(3 \mathrm{H}, \mathrm{s}, \mathrm{CH}_{3}\right), 2.92-2.89(1 \mathrm{H}, \mathrm{m}, \mathrm{CHD}), 2.74\left(2 \mathrm{H}, \mathrm{d}, J=7.91 \mathrm{~Hz}, \mathrm{CH}_{2}\right) ;{ }^{2} \mathrm{H}$ NMR $(77$ $\mathrm{MHz}, \mathrm{DCM}) \delta 9.76(\mathrm{~s}), 2.84(\mathrm{~s}) ;{ }^{13} \mathrm{C} \mathrm{NMR}\left(75.5 \mathrm{MHz}, \mathrm{CDCl}_{3}\right) \delta 158.5,132.7,129.6$, 114.4, 55.7, 54.7, 30.1; HRMS (EI) calcd for $\mathrm{C}_{10} \mathrm{H}_{14} \mathrm{D}_{2} \mathrm{NO}\left[\mathrm{M}+\mathrm{NH}_{4}^{+}\right]: \mathrm{m} / z$ 189.0855, found: $m / z 189.0855$. 


\section{1,2,2,3-tetradeutero-3-(4-methoxyphenyl) propionaldehyde (6a):}

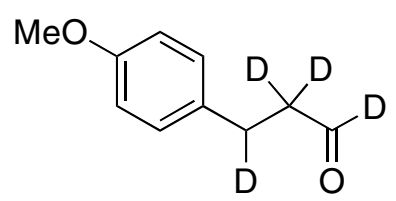

To a solution of 3-(4-methoxyphenyl)-propanal (3a) (0.5 mmol), molybdenum hexacarbonyl (6.6 mg, $5 \mathrm{~mol} \%$ ) and $N$-methylmorpholine- $N$-oxide $(5.9 \mathrm{mg}, 10 \mathrm{~mol} \%)$ in tetrahydrofuran $(3.0 \mathrm{~mL})$ was added trideuteriophenylsilane $(185 \mu \mathrm{L}, 1.5 \mathrm{mmol})$. The resulting solution was stirred under an atmosphere of nitrogen at $80{ }^{\circ} \mathrm{C}$ for $16 \mathrm{~h}$. After cooling to room temperature $\mathrm{D}_{2} \mathrm{O}(0.5 \mathrm{~mL})$ was added and the solution stirred for 15 minutes. The solution was dissolved in ether $(50 \mathrm{~mL})$ was washed with $1 \mathrm{M} \mathrm{NaOH}(3 \mathrm{x}$ $50 \mathrm{~mL})$ followed by brine $(2 \times 50 \mathrm{~mL})$. The organic phase was extracted, dried over $\mathrm{MgSO}_{4}$ and concentrated in vacuo. The desired product was isolated by flash column chromatography (petrol : ethyl acetate 11:1) as a colourless oil (66.5 mg, $81 \%) ; 97 \%$ deuterium incorportation; $v_{\max }$ (neat) $/ \mathrm{cm}^{-1} ; 2838(\mathrm{C}-\mathrm{D}) ; 1709$ (C=O); $1246(\mathrm{C}-\mathrm{O}) ;{ }^{1} \mathrm{H}$ NMR (300 MHz, CDCl $) \delta 7.12(2 \mathrm{H}, \mathrm{d}, J=8.67 \mathrm{~Hz}, \mathrm{Ar}), 6.84(2 \mathrm{H}, \mathrm{d}, J=8.67 \mathrm{~Hz}, \mathrm{Ar})$, $3.79\left(3 \mathrm{H}, \mathrm{s}, \mathrm{CH}_{3}\right), 2.87(1 \mathrm{H}, \mathrm{s}, \mathrm{CHD}){ }^{2} \mathrm{H}$ NMR $(77 \mathrm{MHz}, \mathrm{DCM}) \delta 9.76(\mathrm{~s}, \mathrm{CDO}), 2.81$ $(\mathrm{d}, J=2.1 \mathrm{~Hz}, \mathrm{CDH}) 2.60\left(\mathrm{~s}, \mathrm{CD}_{2}\right) ;{ }^{13} \mathrm{C} \mathrm{NMR}\left(75.5 \mathrm{MHz}, \mathrm{CDCl}_{3}\right) \delta 158.5,132.7,129.6$, 114.4, 55.7, $27.2\left(\mathrm{t}, J=19.85 \mathrm{~Hz}\right.$ ); HRMS (EI) calcd for $\mathrm{C}_{10} \mathrm{H}_{8} \mathrm{D}_{4} \mathrm{O}_{2} \mathrm{Na}[\mathrm{M}+\mathrm{Na}]^{+}: \mathrm{m} / \mathrm{z}$ 191.0981, found: $\mathrm{m} / \mathrm{z} 191.0981$. 

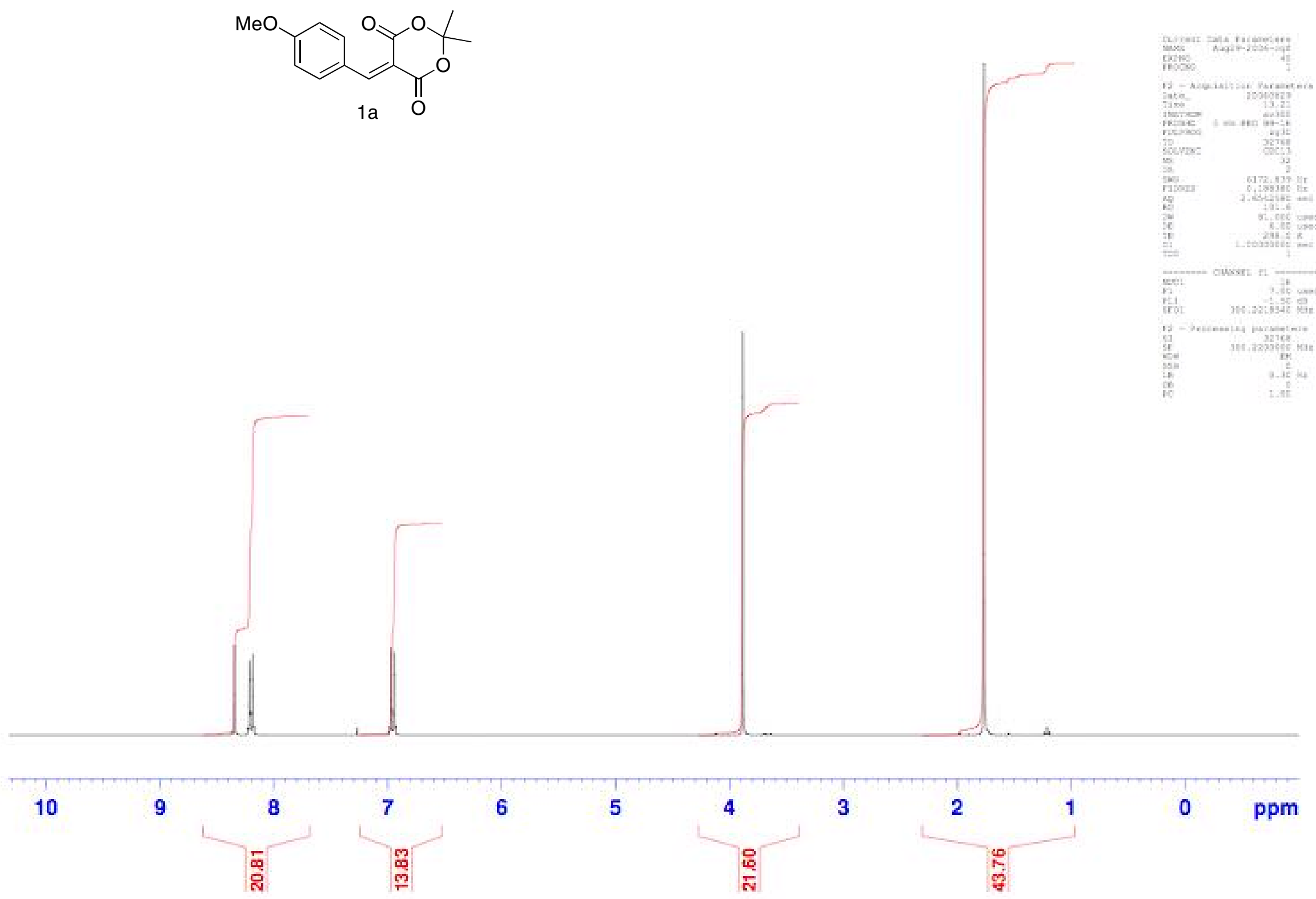
<smiles>COc1ccc(C=C2C(=O)OC(C)(C)OC2=O)cc1</smiles>
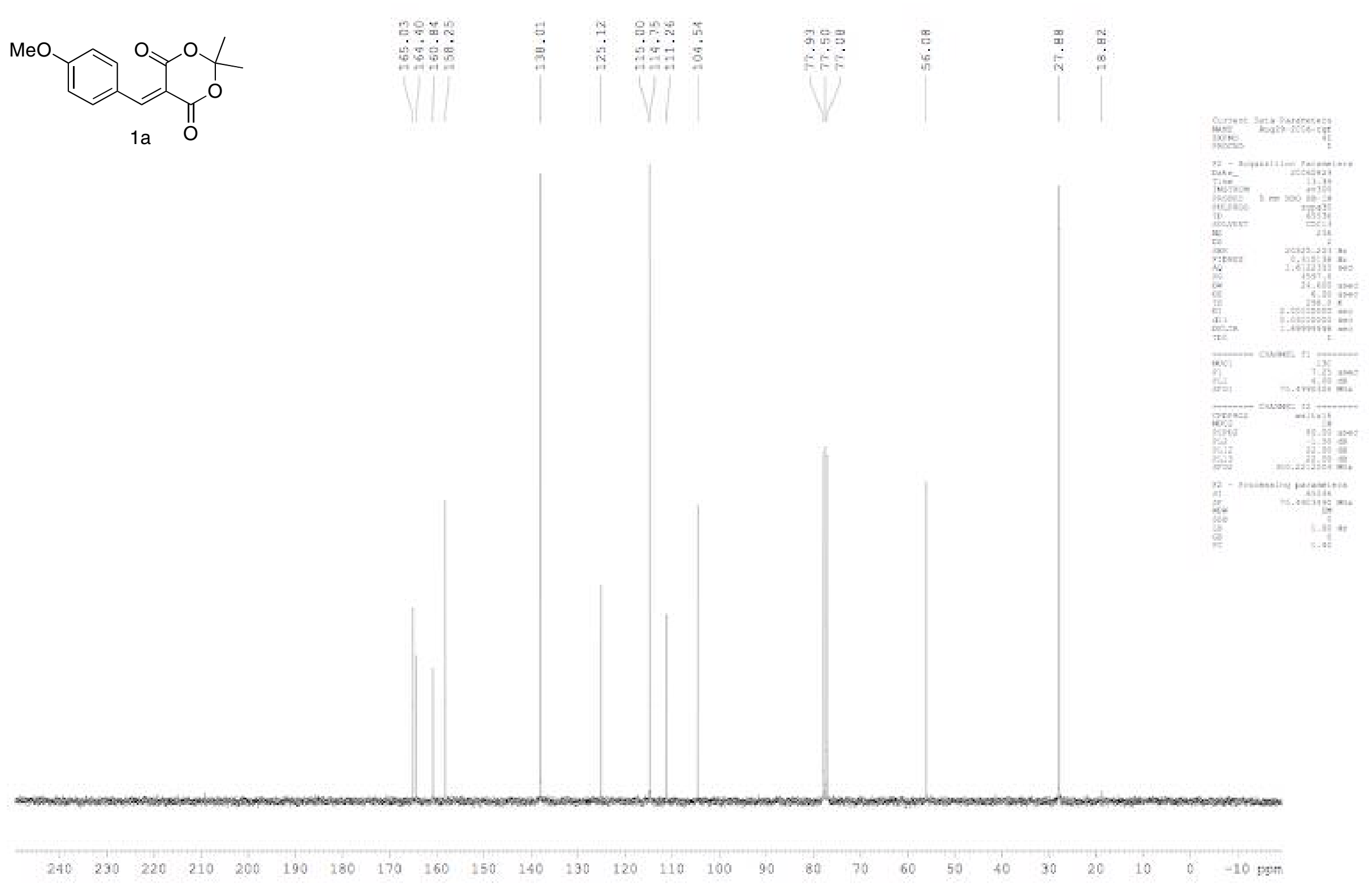
$\mathrm{Me}_{2} \mathrm{~N}$

1b

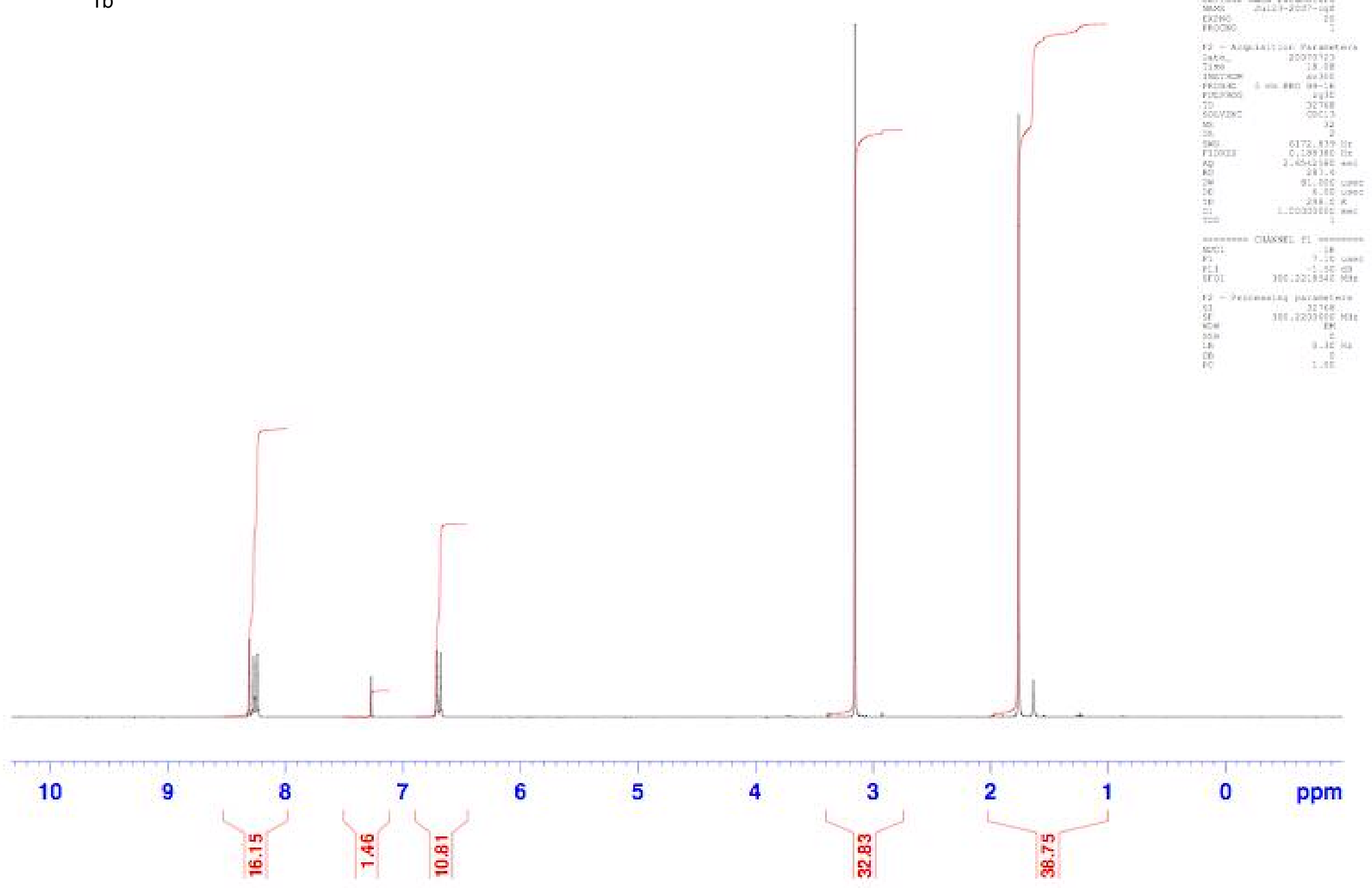


$\mathrm{Me}_{2} \mathrm{~N} \mathrm{~T}_{\mathrm{O}}^{\mathrm{O}}$

$1 \mathrm{~b}$

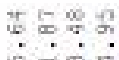

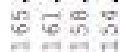

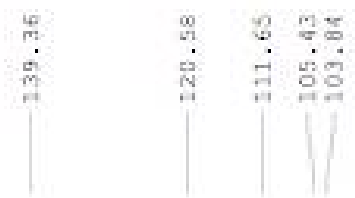

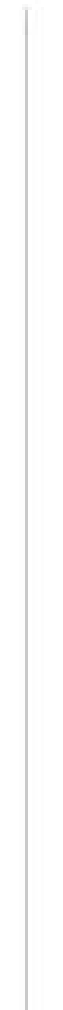

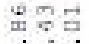 \\ Fin}

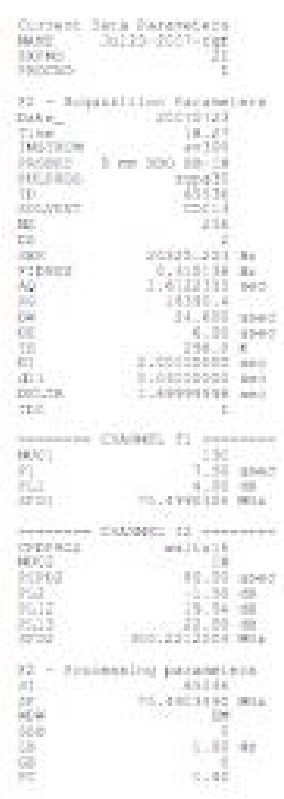

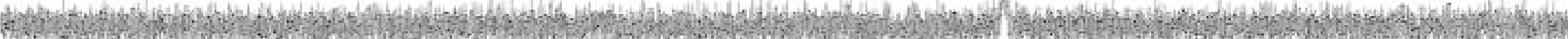
$240 \quad 23$
220
10. 200
280
1016
$50 \quad 140$
$130 \quad 120$
$110 \quad 100$
80
o 60
50
$40 \quad 30$
$20 \quad 10$
$-10 \mathrm{pgm}$ 
SDP306 - 4-SMe Phenyl Meldrum's Acid Condensed

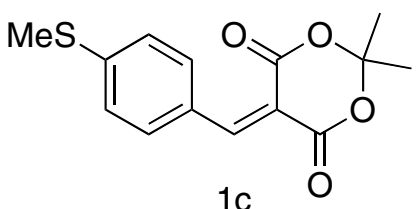

$1 c$

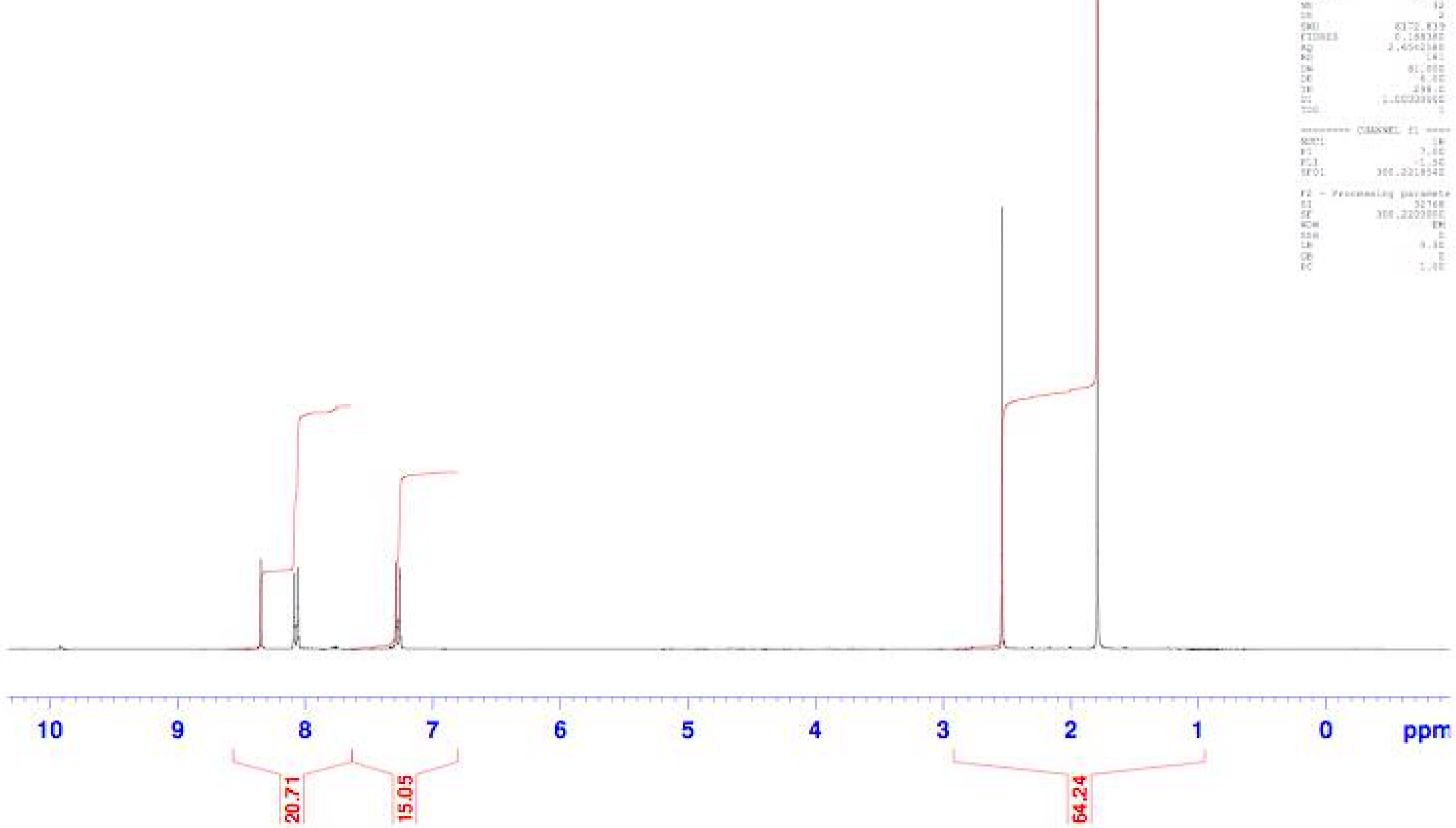


$\mathrm{MeS}_{\mathrm{O}}^{\mathrm{M}}$

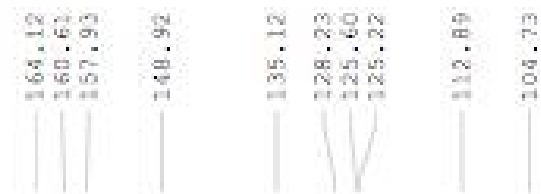

묵응

$\stackrel{g}{\square}:$

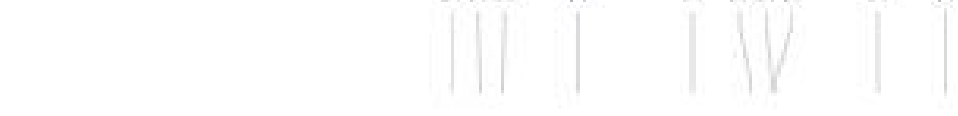

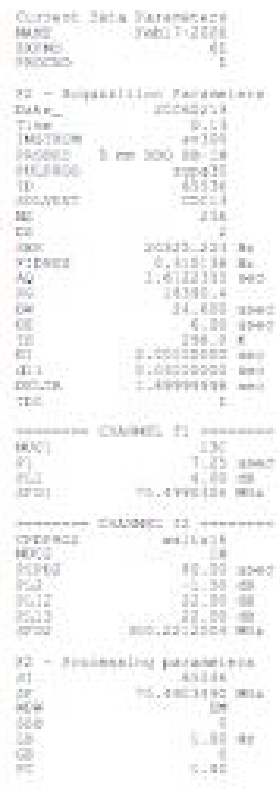

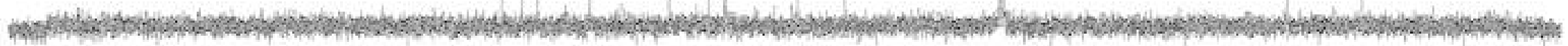

$\begin{array}{lllllllllllllllllllllllllllll}240 & 230 & 220 & 210 & 200 & 190 & 280 & 170 & 160 & 150 & 140 & 130 & 120 & 110 & 100 & 90 & 80 & 70 & 60 & 50 & 40 & 30 & 20 & 10 & 0 & -10 & \text { pam }\end{array}$ 


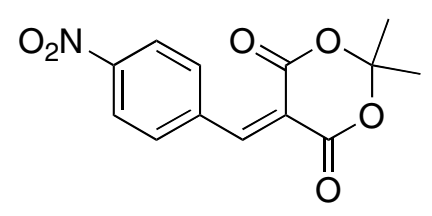

1d

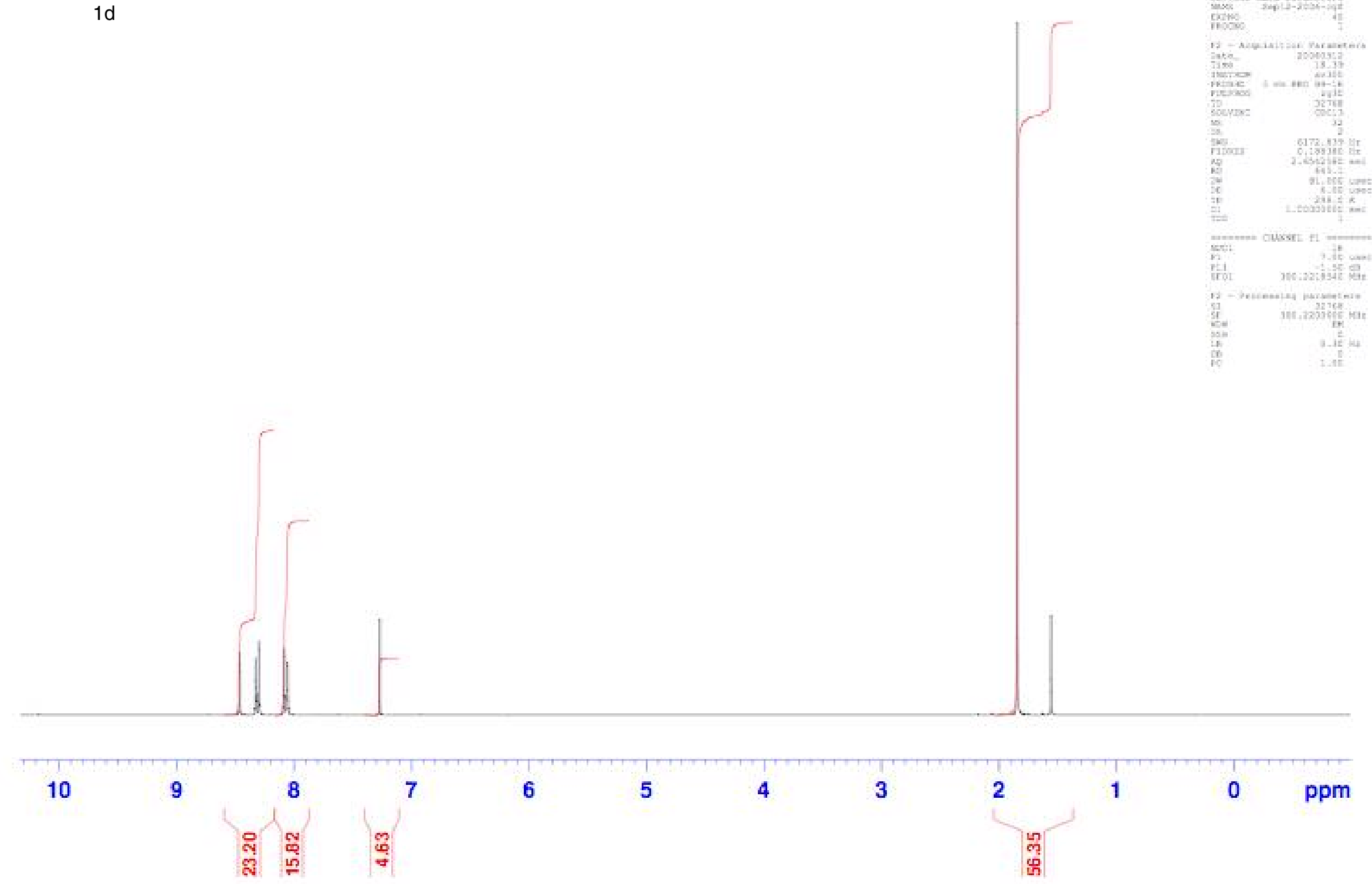




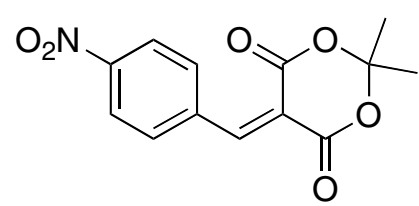

$1 d$

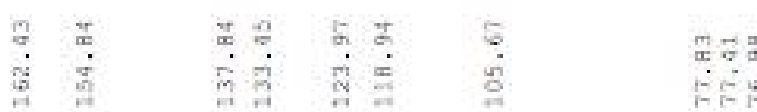

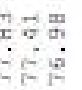

$\stackrel{n}{\stackrel{n}{*}}$

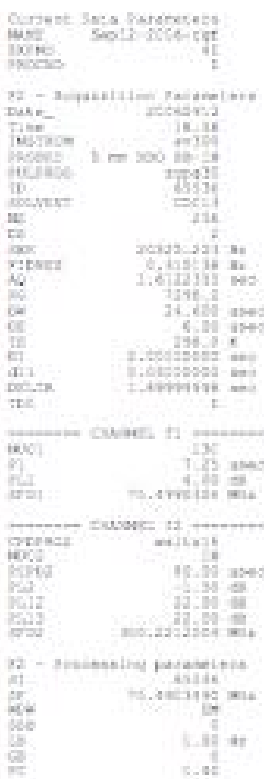

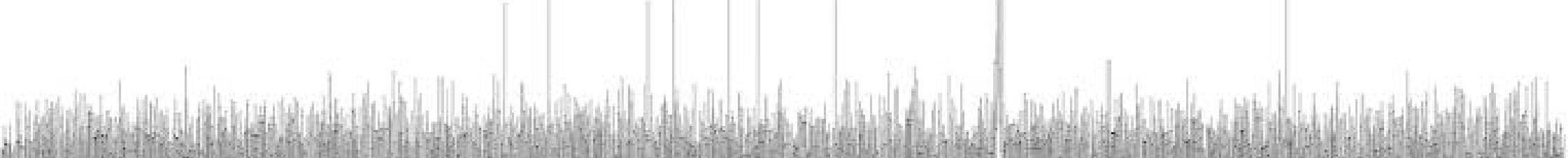
An

$\begin{array}{lllllllllllllllllllllllllllll}240 & 290 & 220 & 210 & 200 & 190 & 280 & 170 & 160 & 150 & 140 & 130 & 120 & 110 & 100 & 90 & 80 & 70 & 60 & 59 & 40 & 30 & 20 & 10 & 0 & -10 & 59 m\end{array}$ 


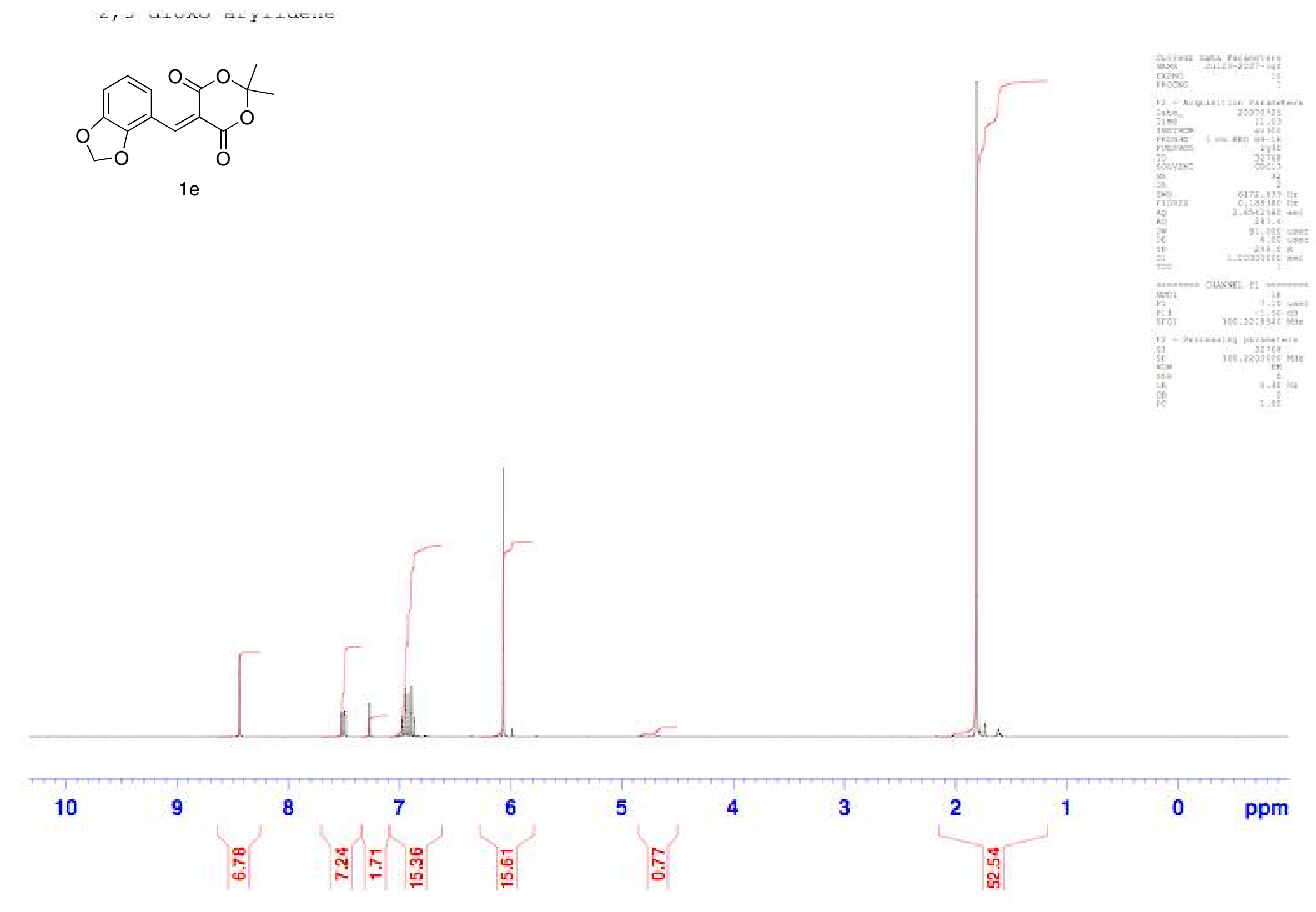



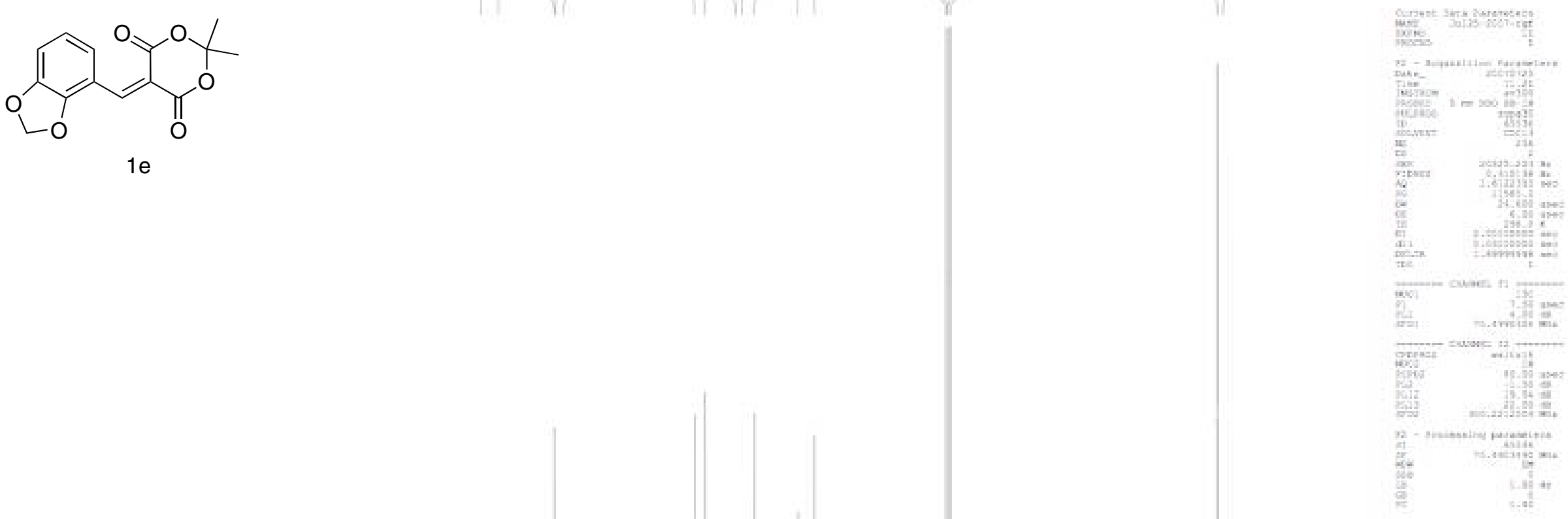

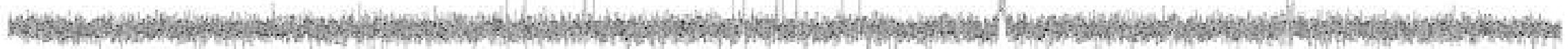
$240 \quad 230$
$220 \quad 210 \quad 200$
190
$170 \quad 160$
$150 \quad 140$
$130 \quad 120$
$110 \quad 100$
80
60
5.
30
10
$-10 \mathrm{pgm}$ 


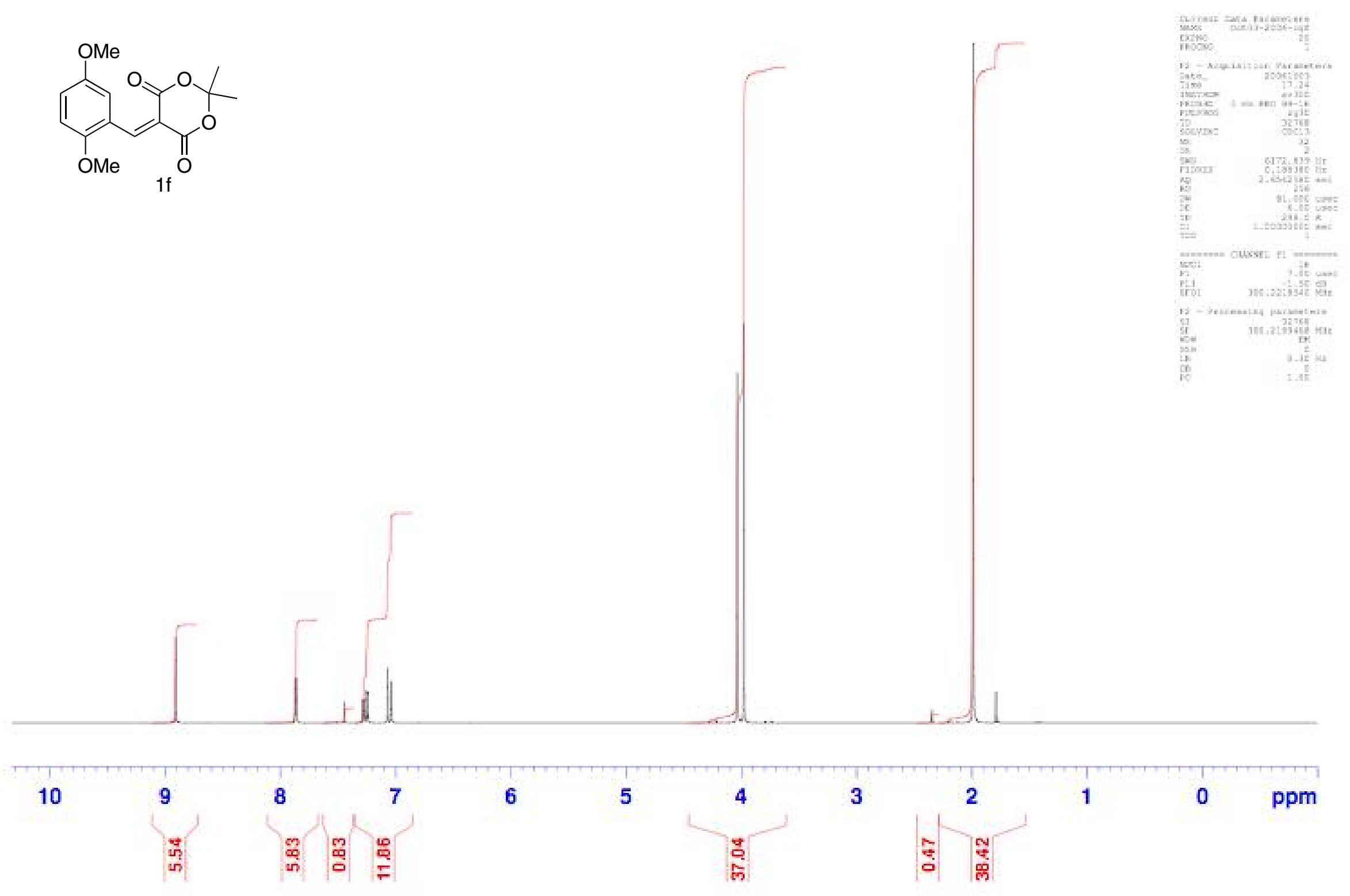



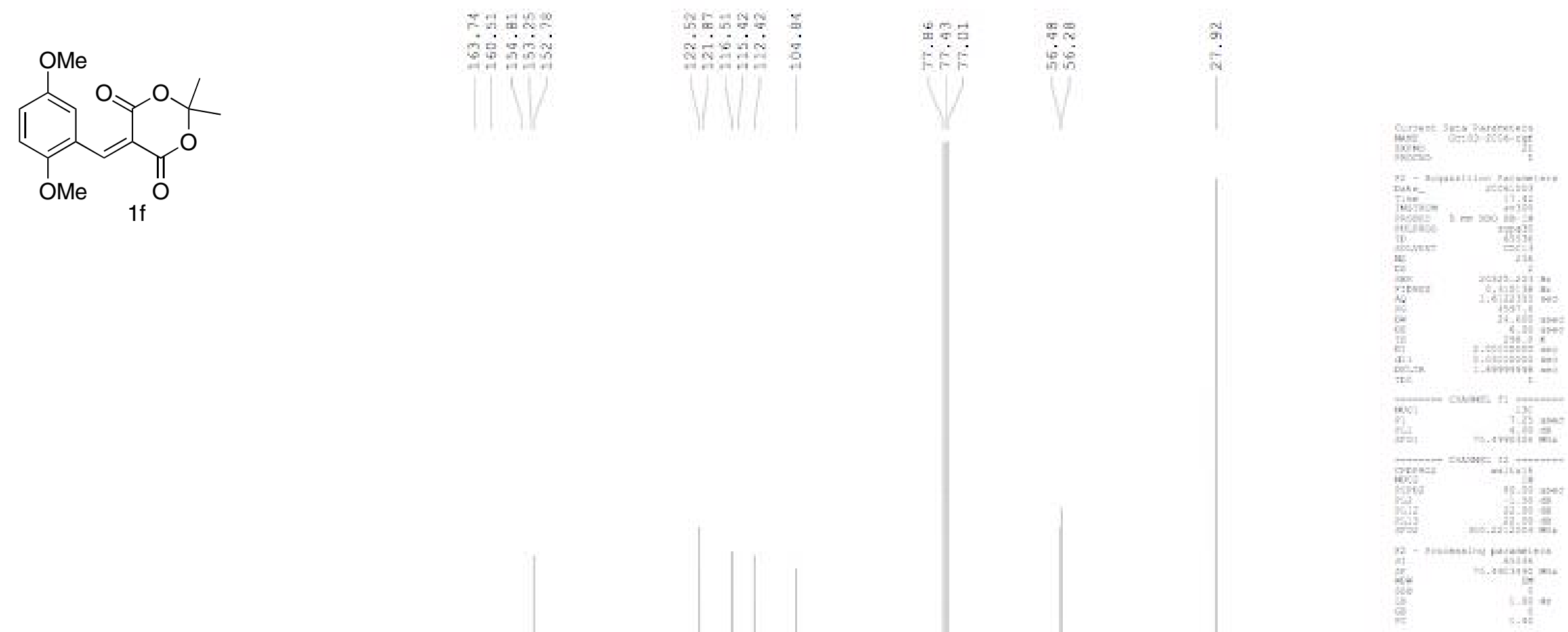

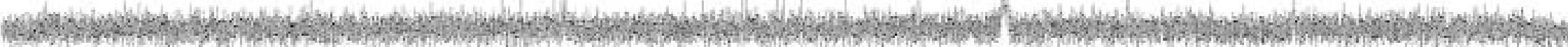

$\begin{array}{lllllllllllllllllllllllllllll}240 & 230 & 220 & 210 & 200 & 190 & 280 & 170 & 160 & 150 & 140 & 130 & 120 & 110 & 100 & 90 & 80 & 70 & 60 & 50 & 40 & 30 & 20 & 10 & 0 & -10 & \text { pam }\end{array}$ 

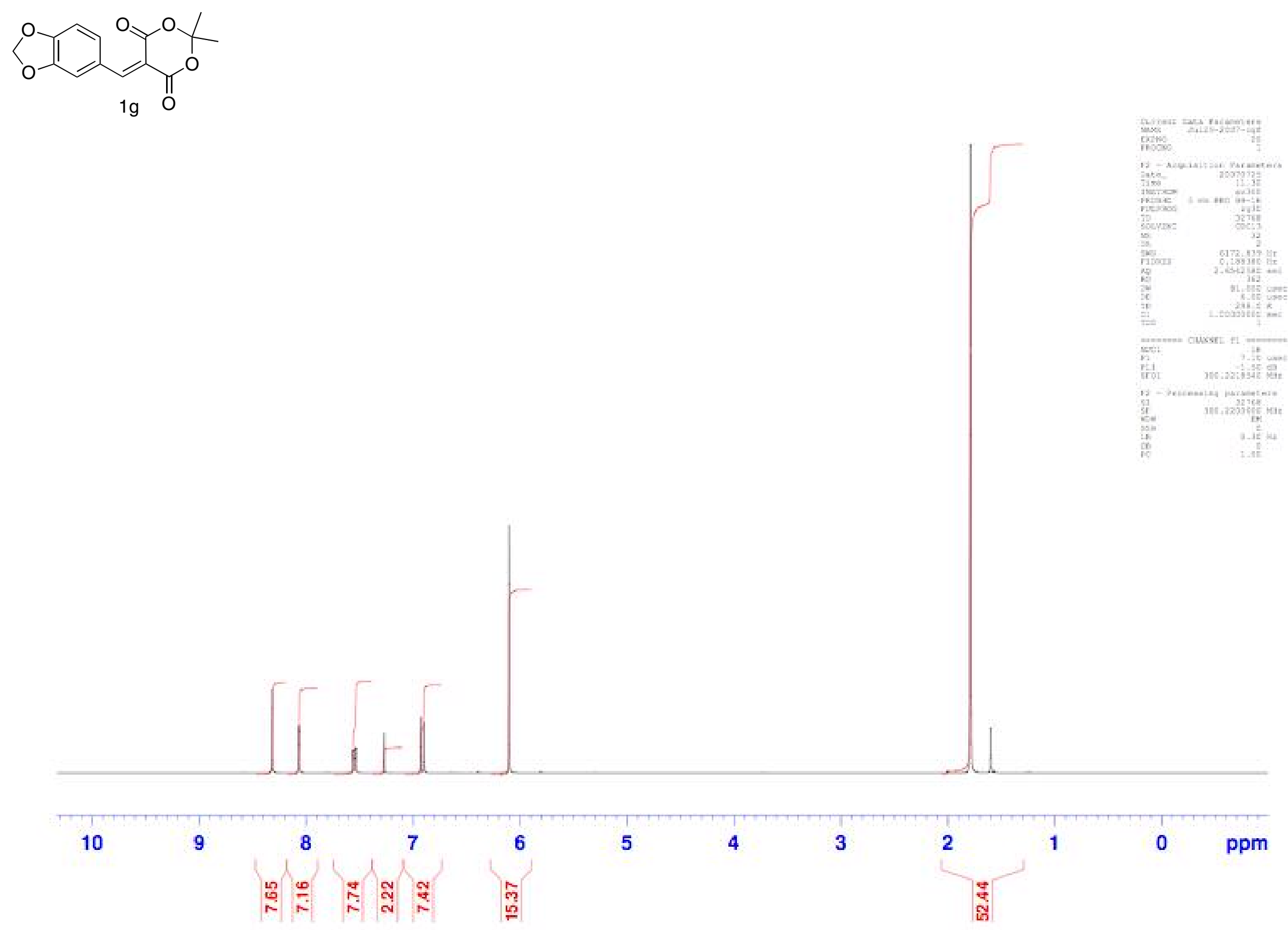


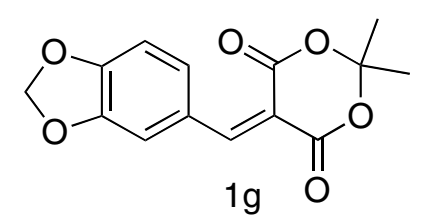

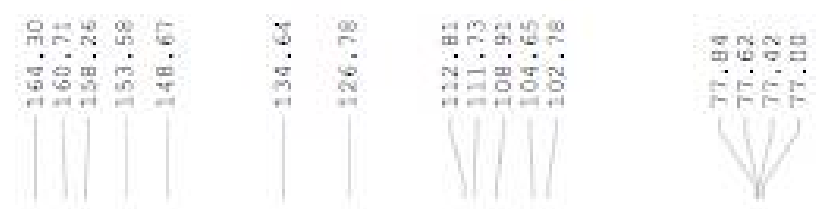

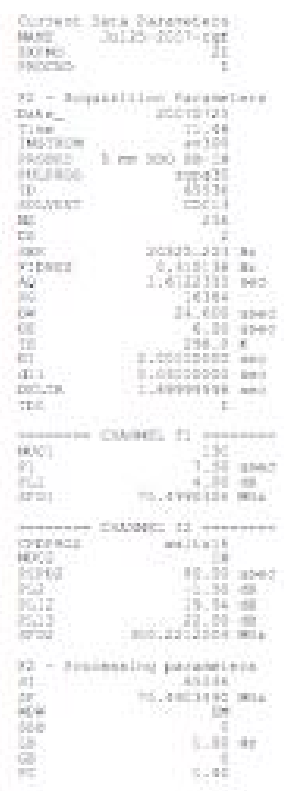

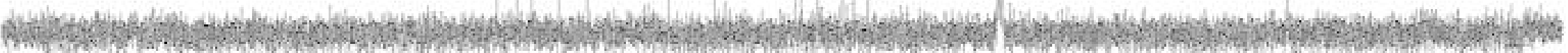

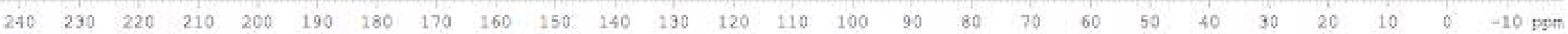




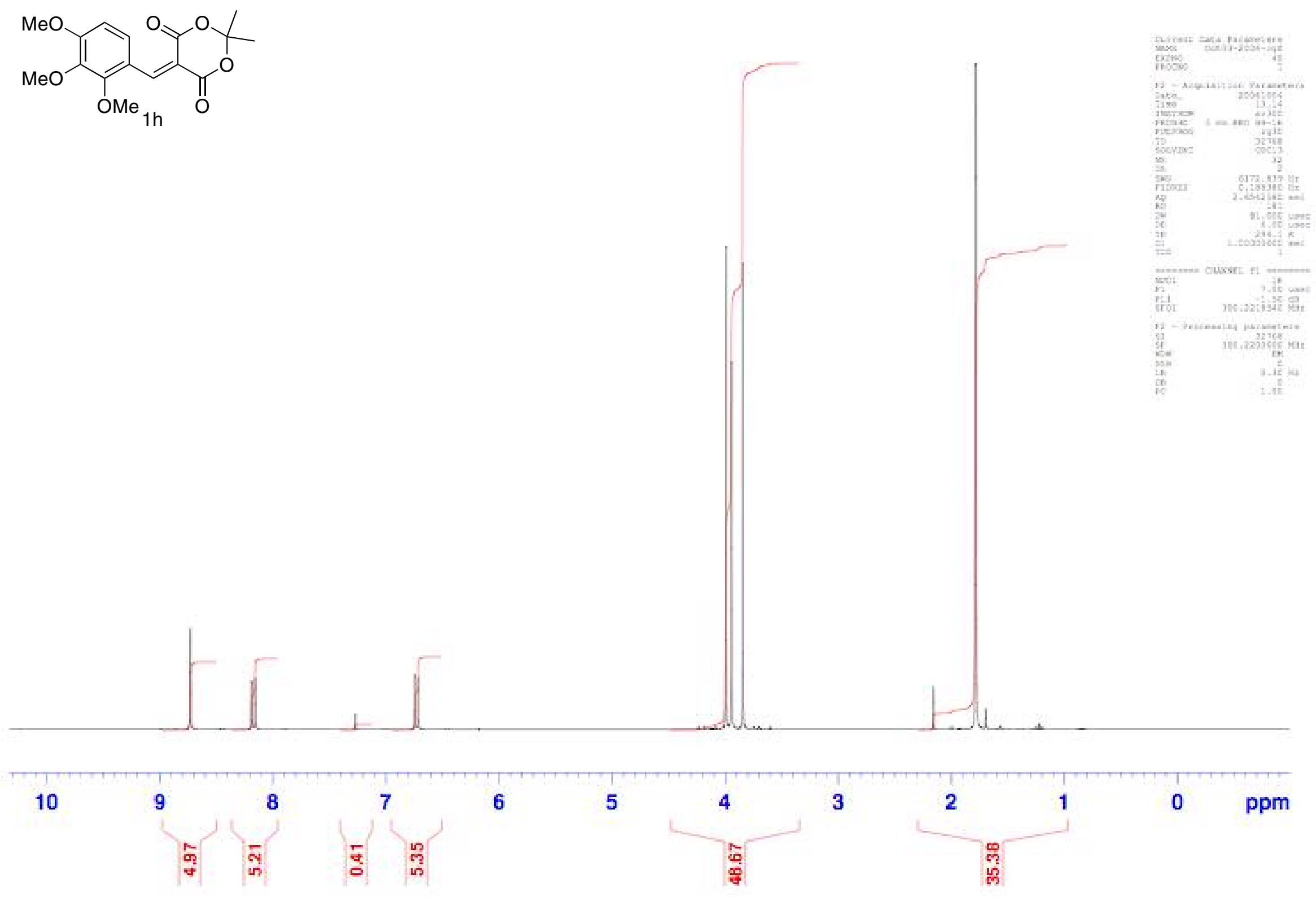



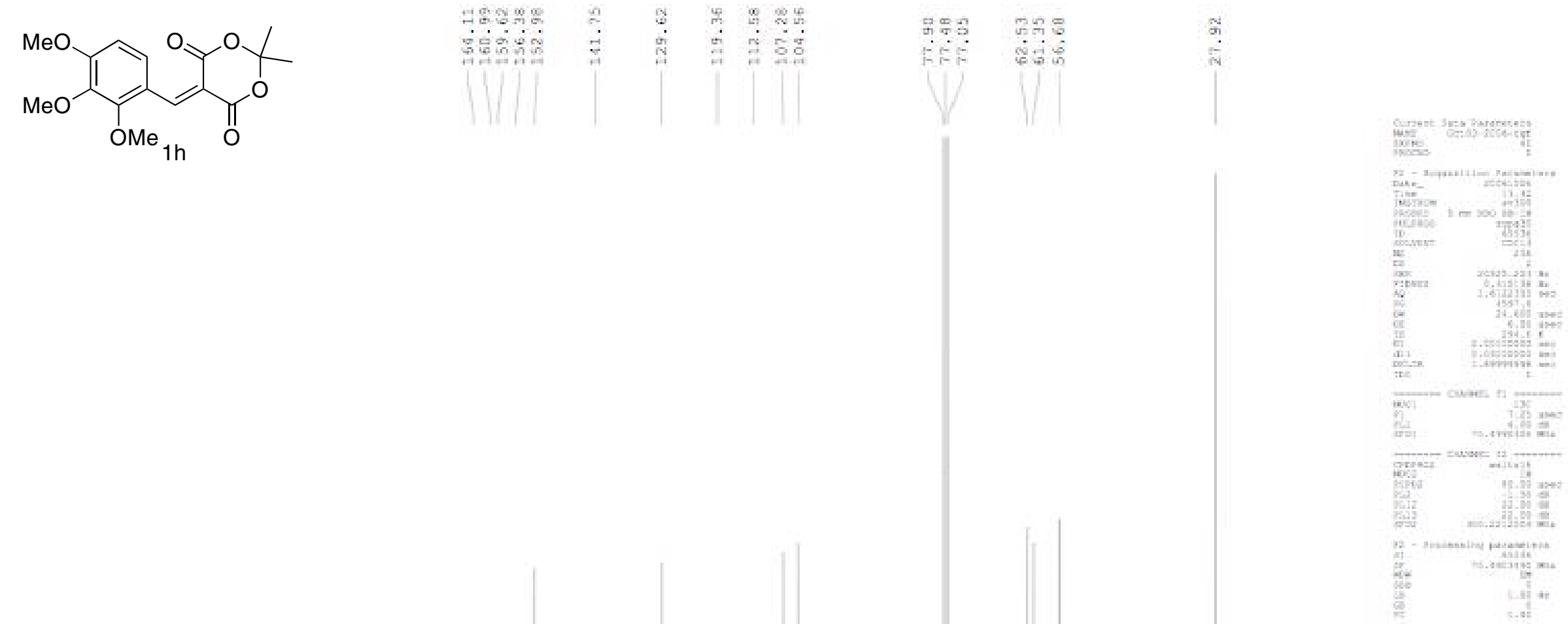

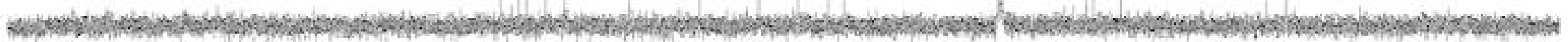



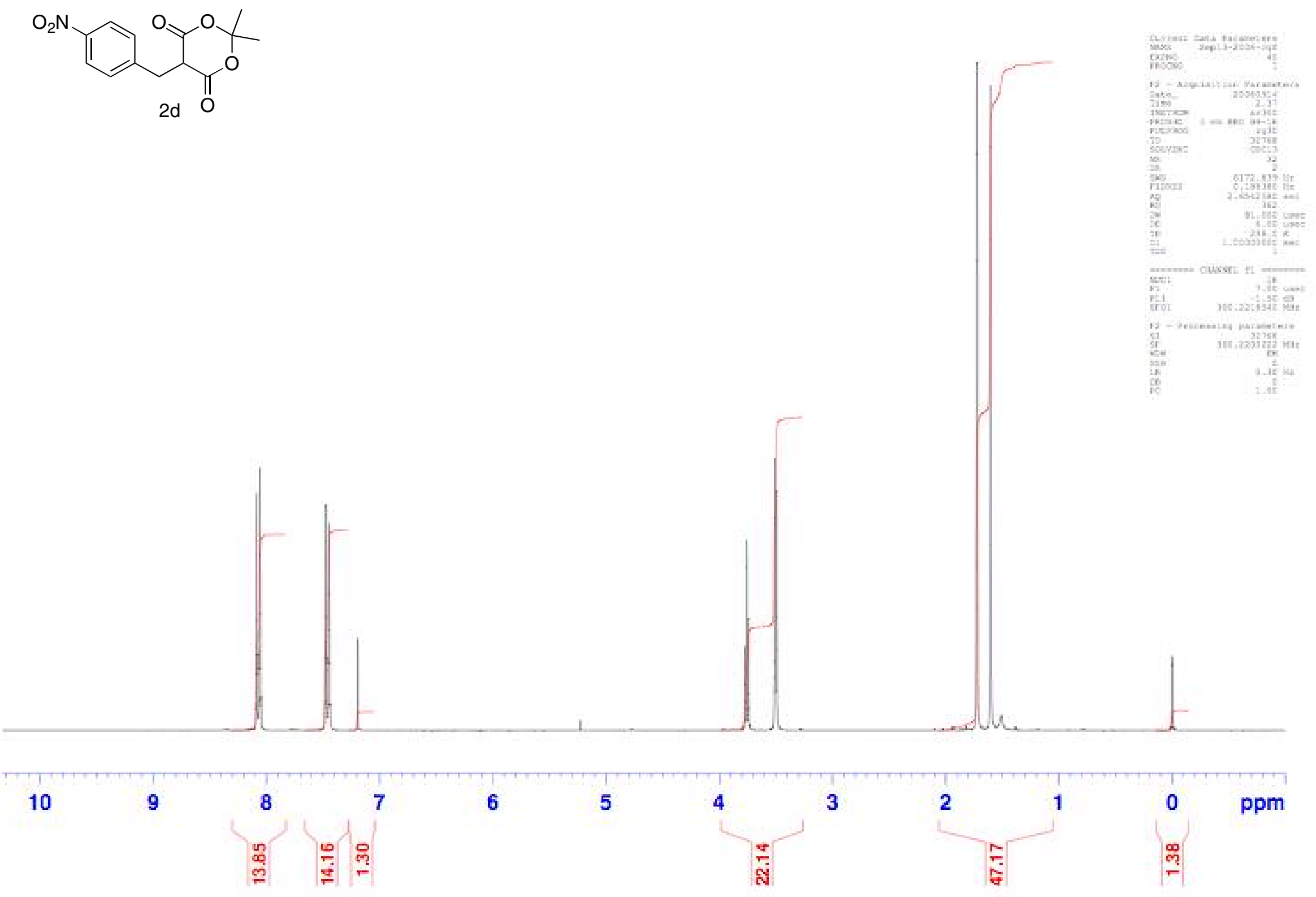

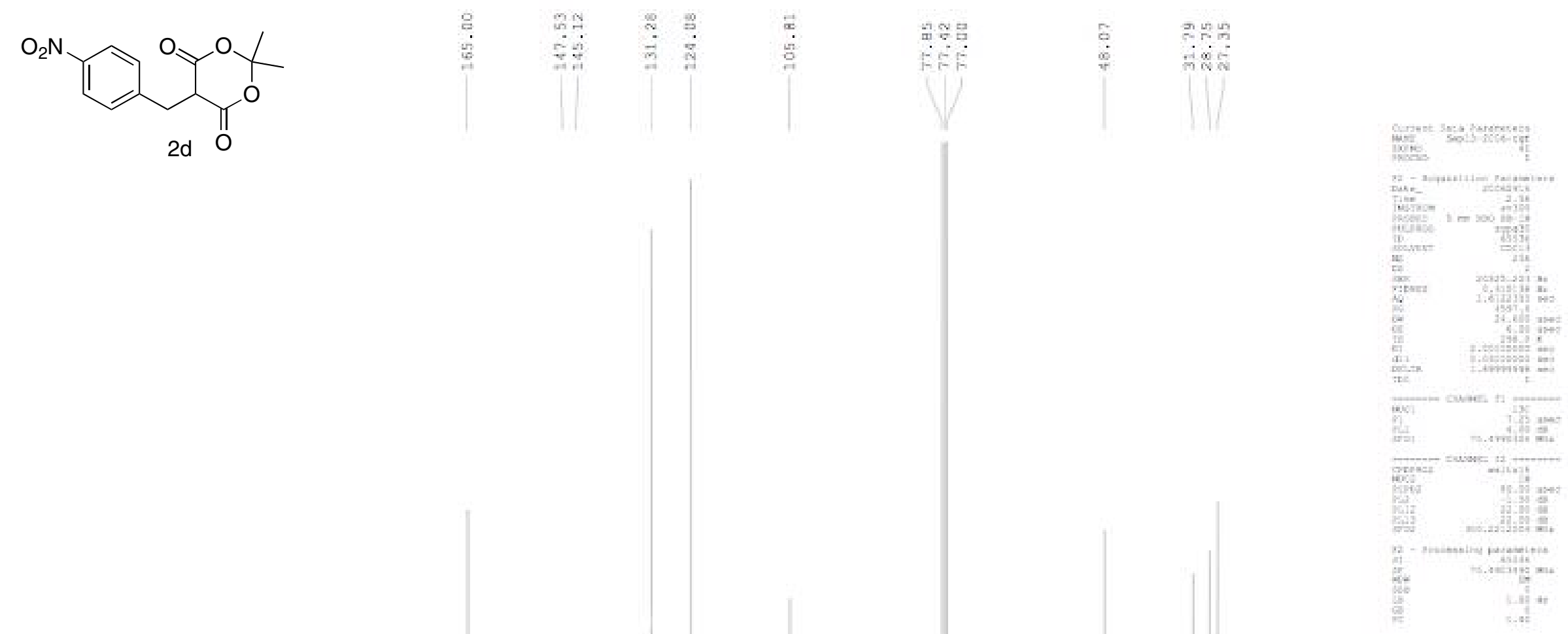

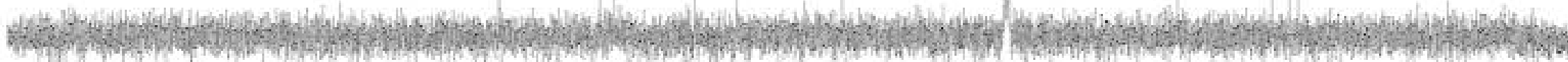

$\begin{array}{lllllllllllllllllllllllllll}240 & 230 & 220 & 210 & 200 & 190 & 280 & 170 & 160 & 150 & 140 & 130 & 120 & 110 & 100 & 90 & 80 & 70 & 60 & 50 & 40 & 30 & 20 & 10 & 0 & -10 & \text { pem }\end{array}$ 


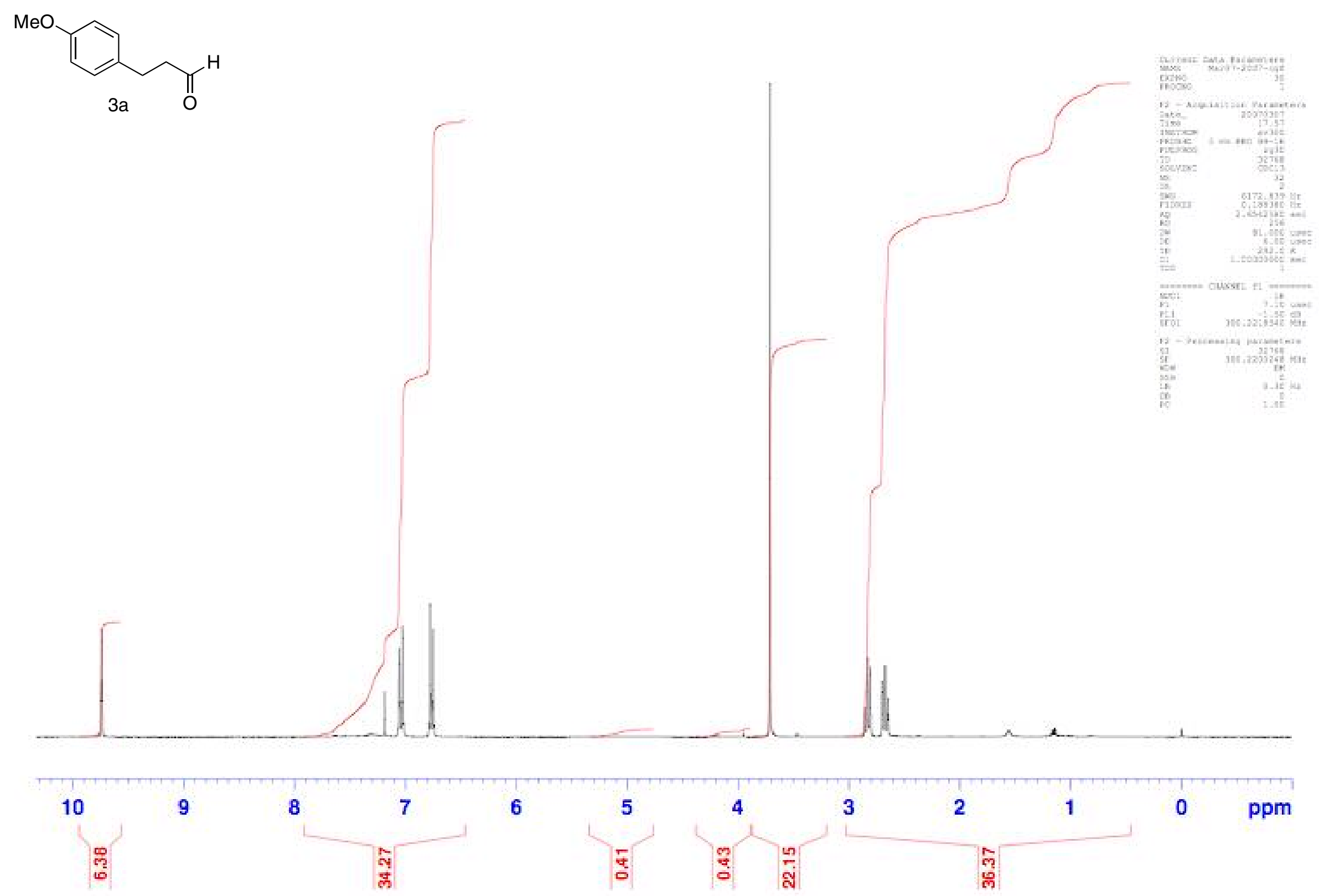




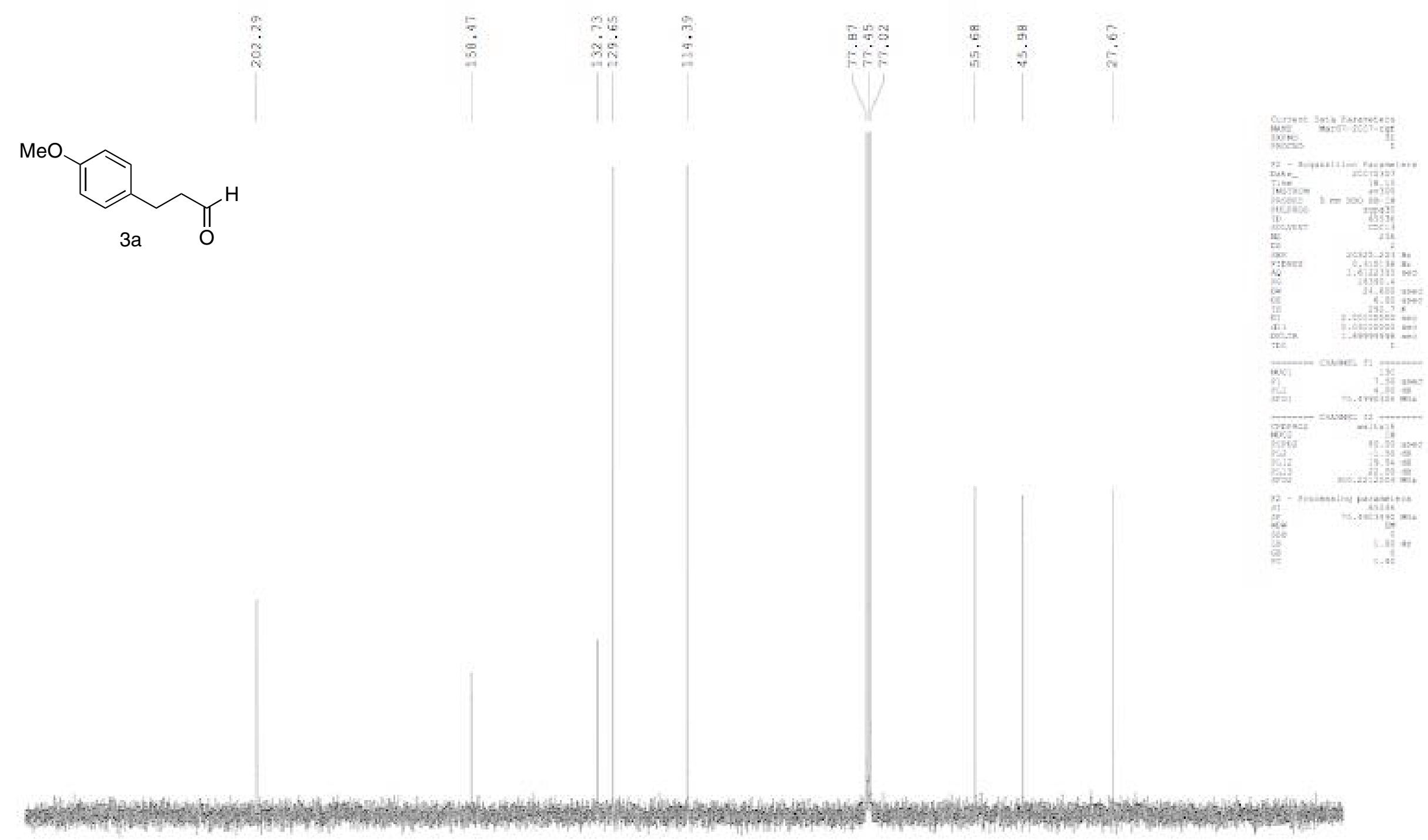

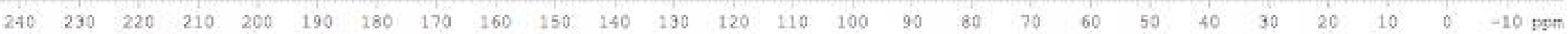


$\mathrm{Me}_{2} \mathrm{~N}$

$3 b$
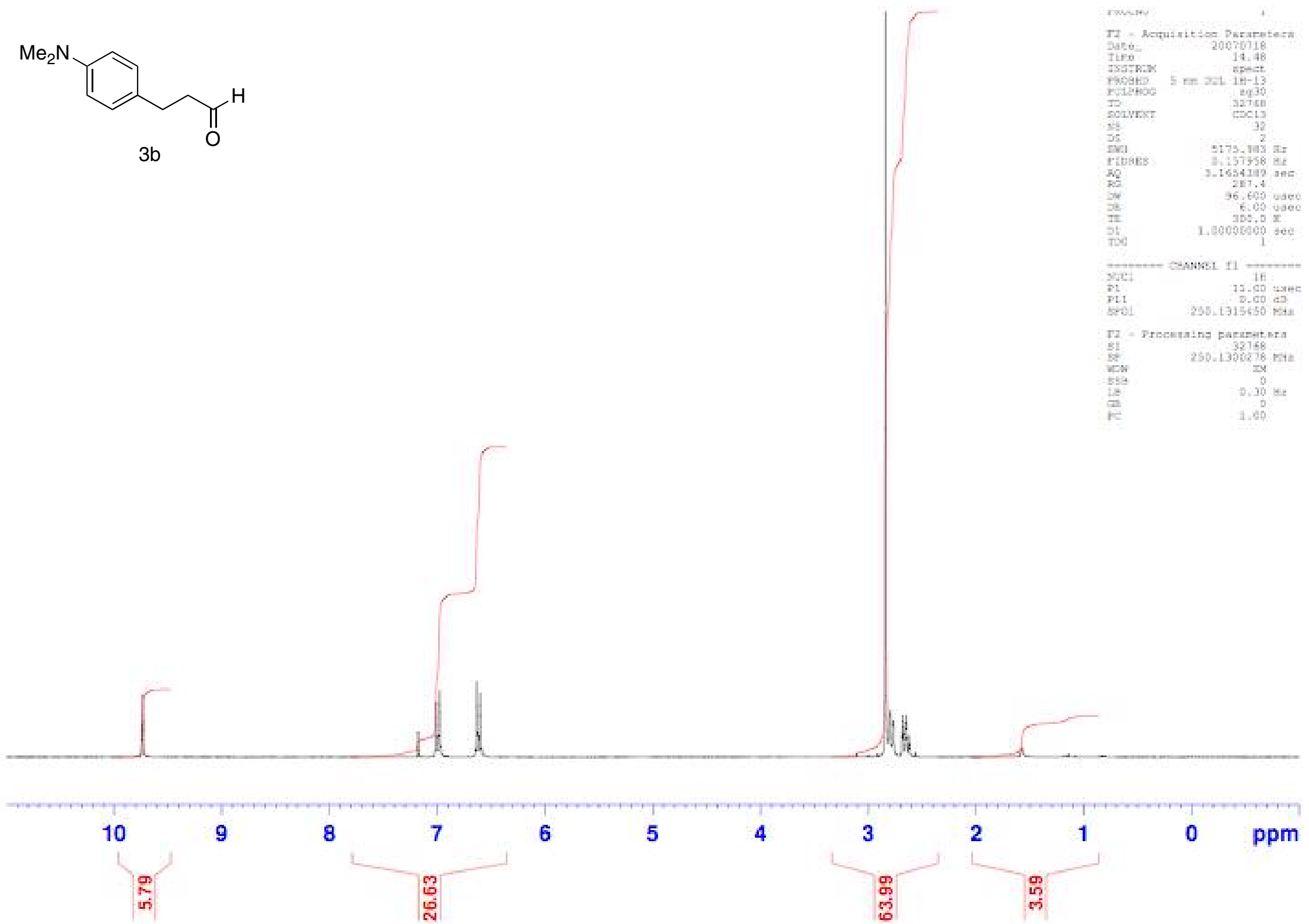


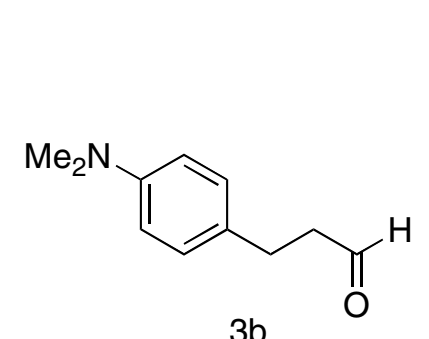

要

$3 b$

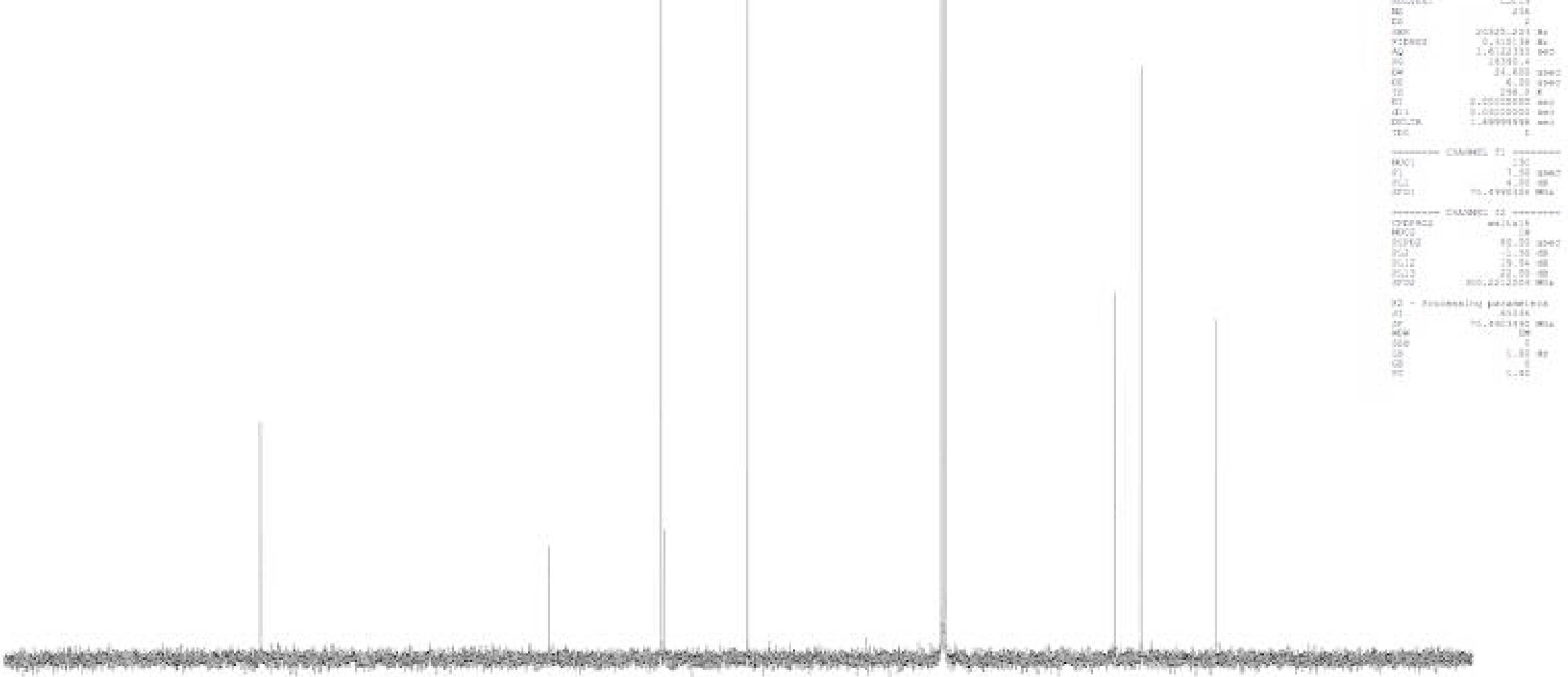




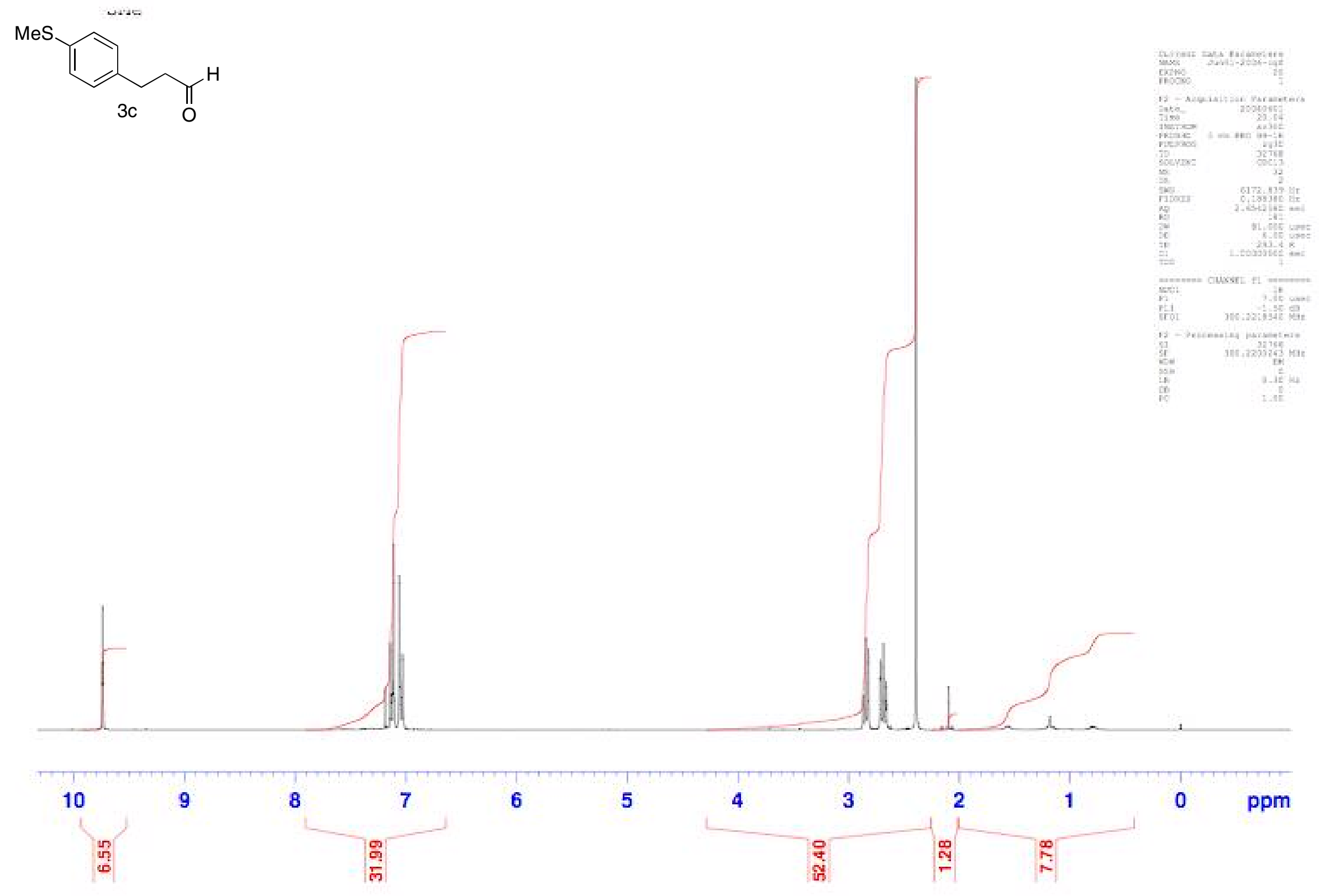




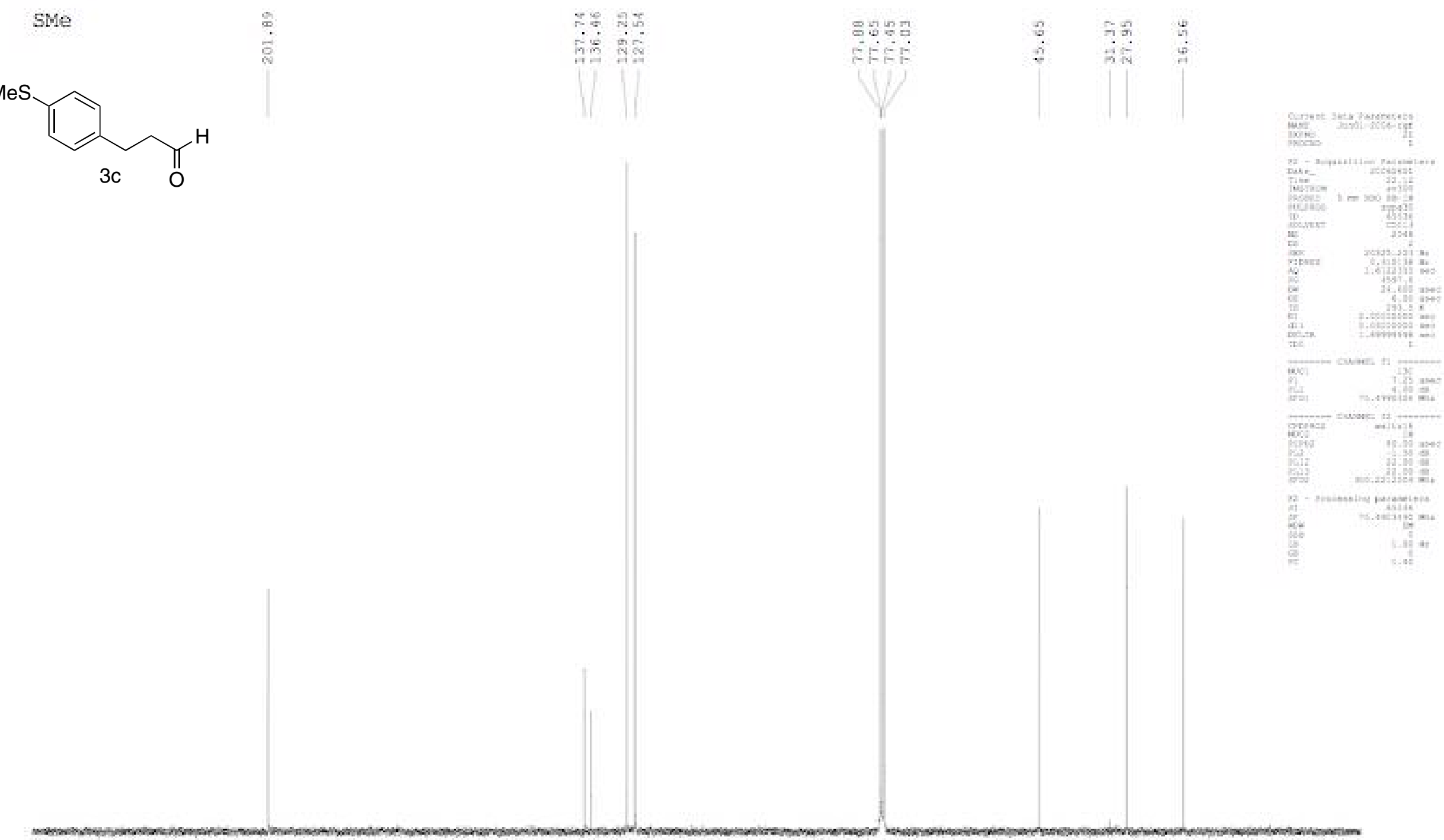

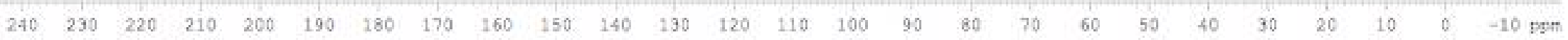




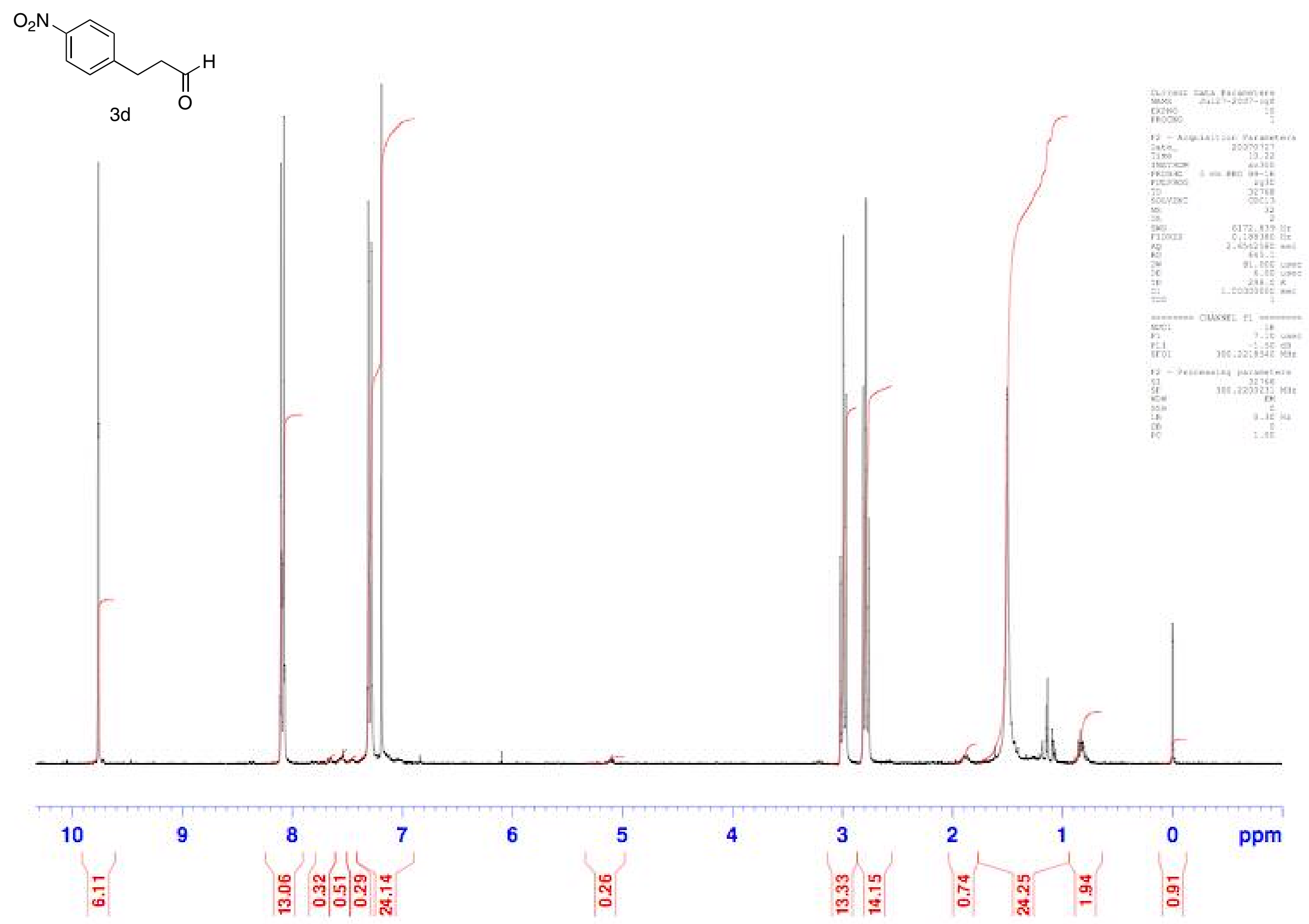


$\mathrm{O}_{2} \mathrm{~N}$<smiles>CC(=O)CCc1ccc(I)cc1</smiles>

3d

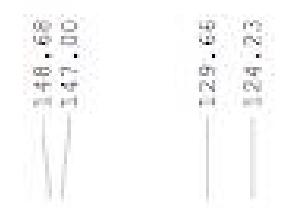

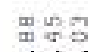

$\stackrel{5}{\dot{3}}$

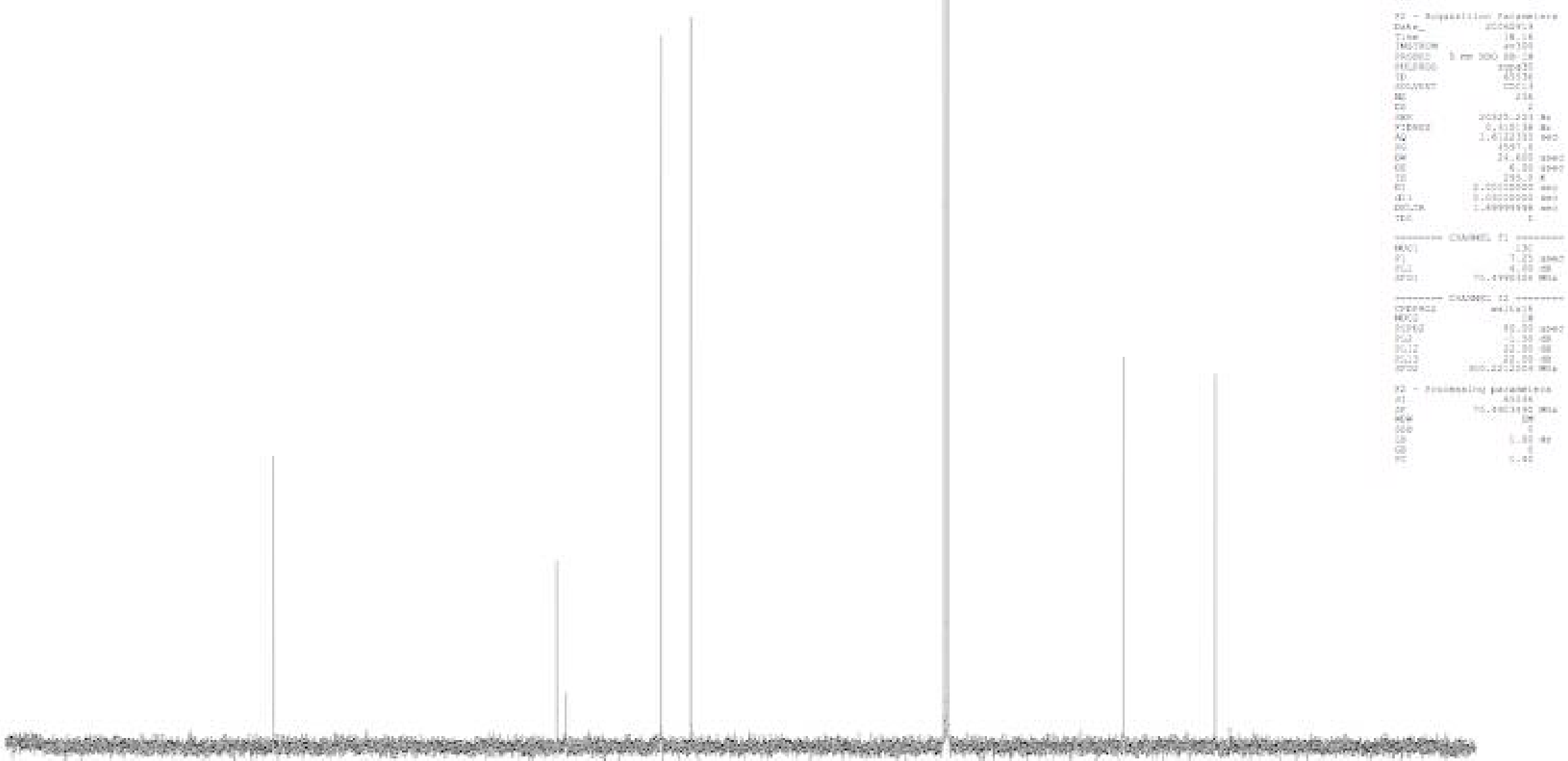


$\underbrace{1}_{3 e}$
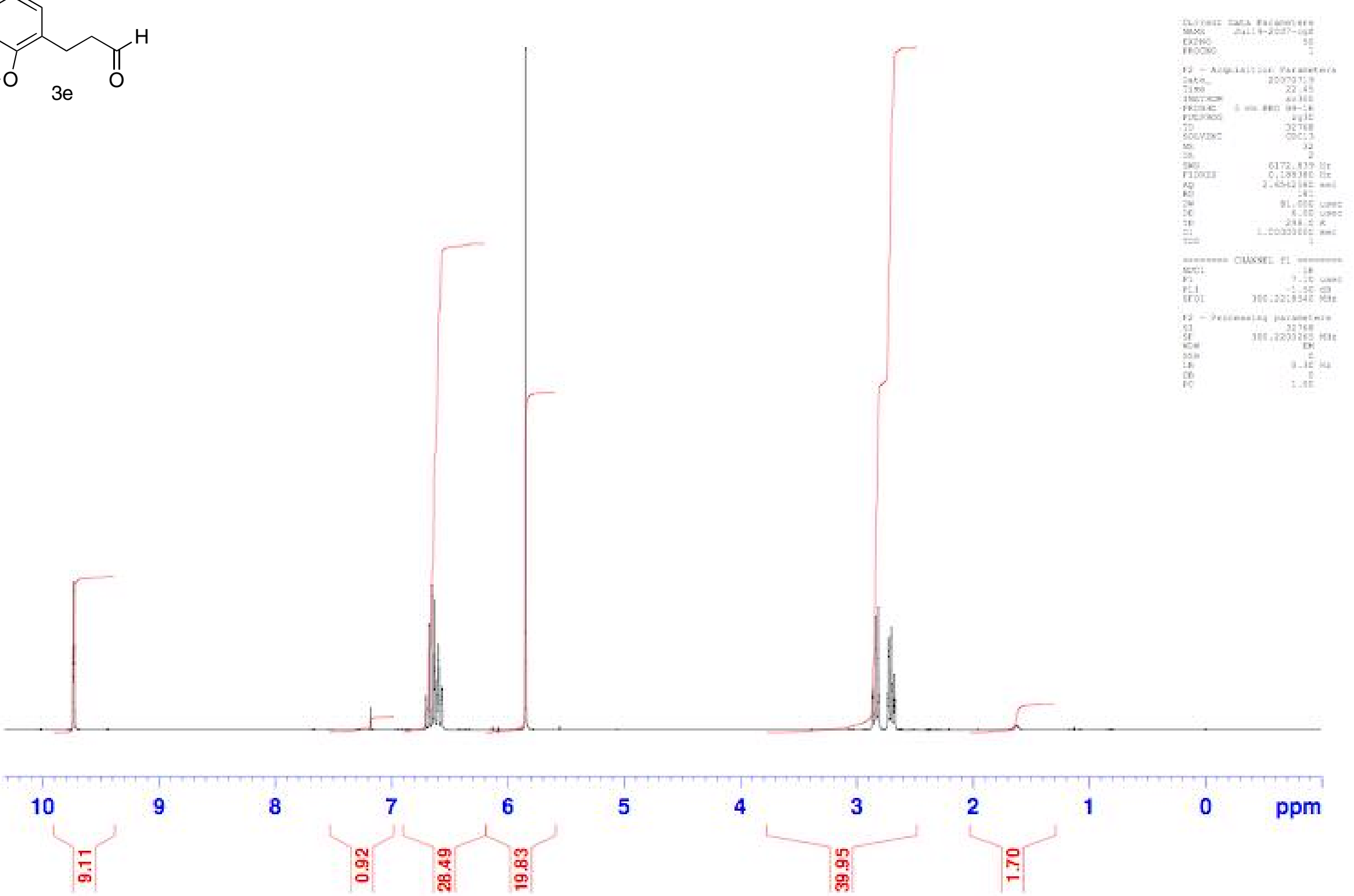

3

क्
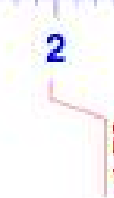

$\underbrace{1}_{3 e}$

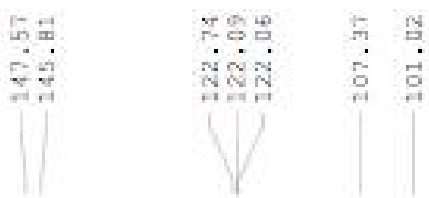
出宁品
ชं

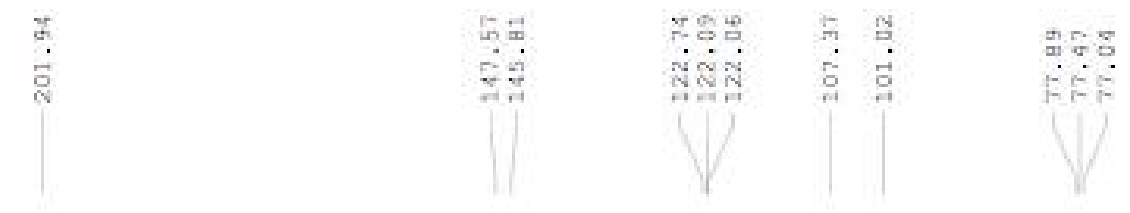

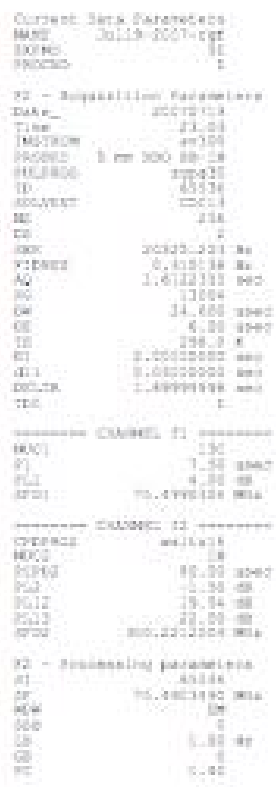

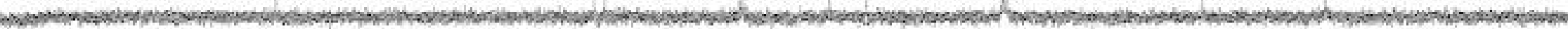



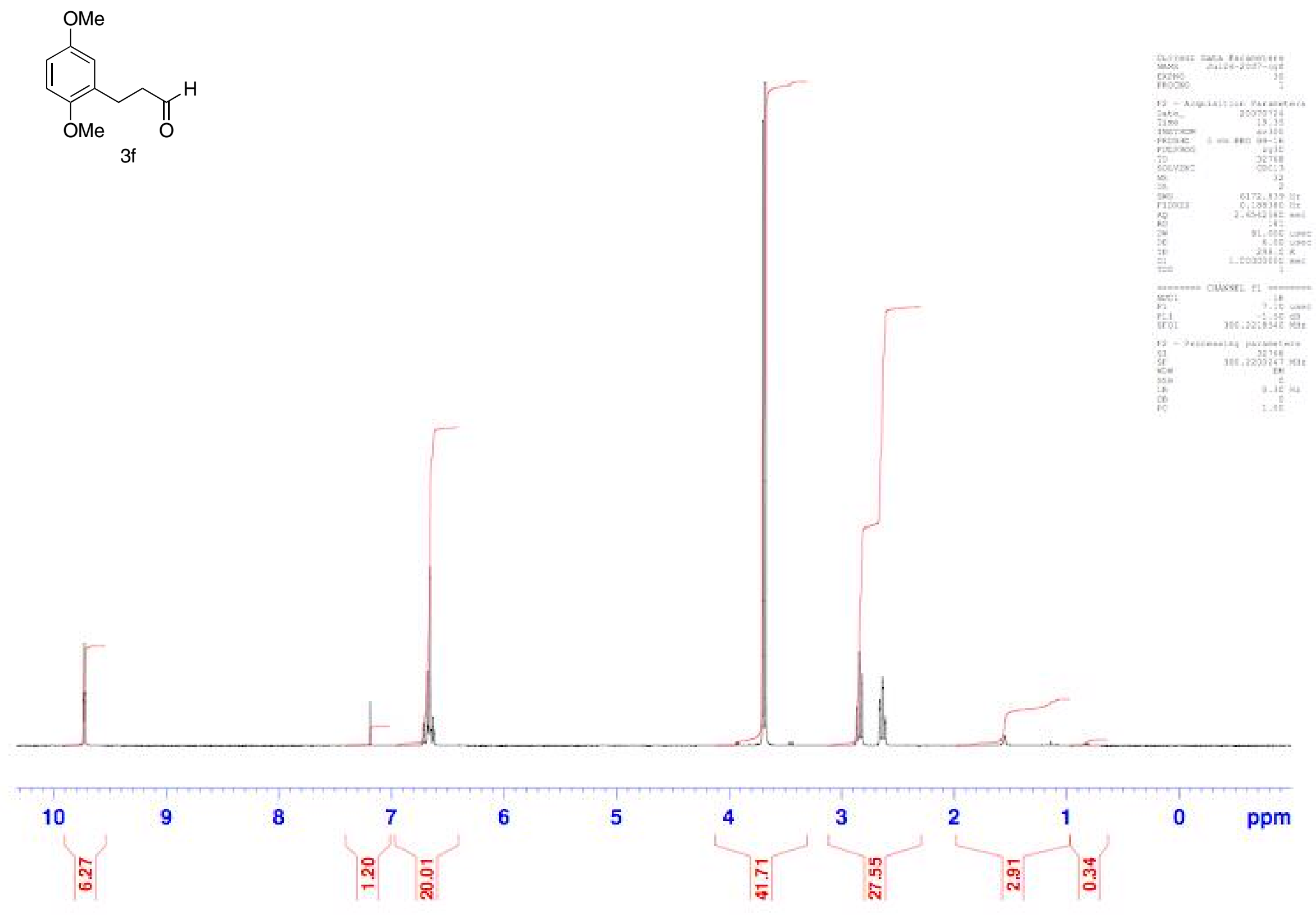


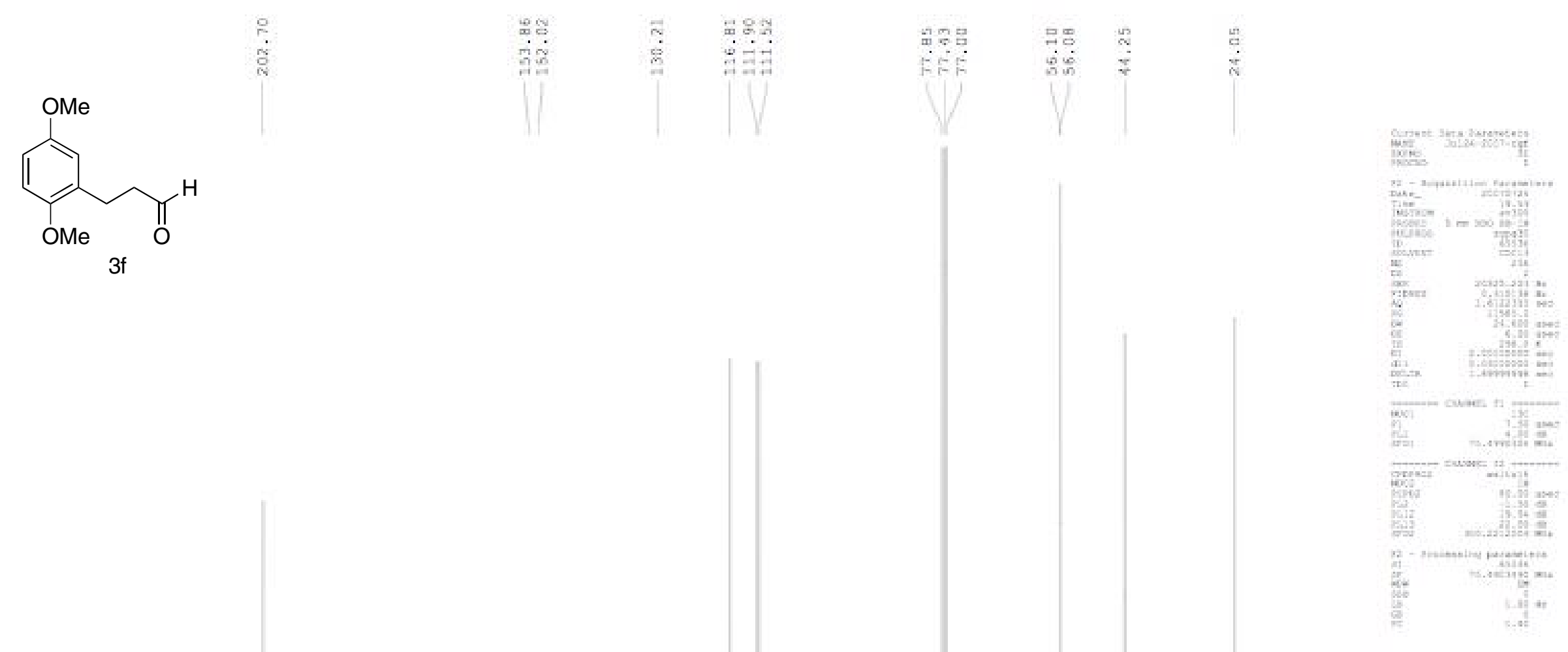

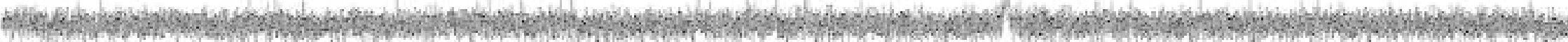

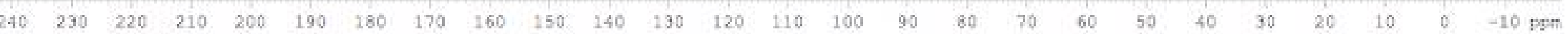




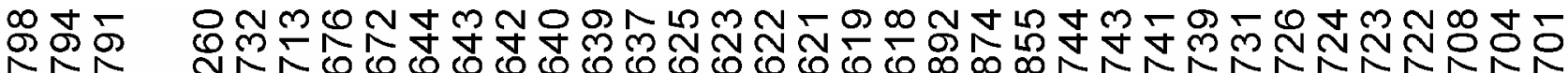

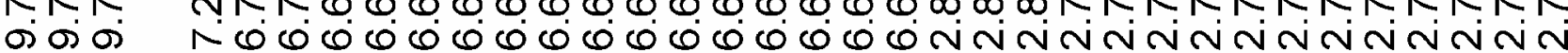

i

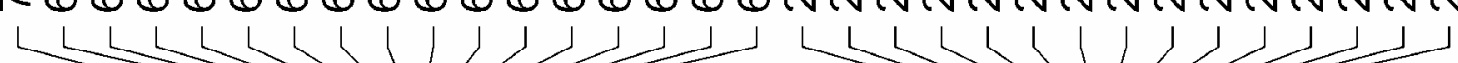
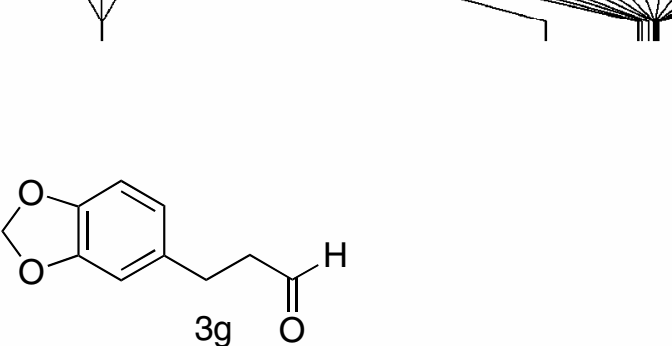

11

Our $r$

NAME

EXPNO

PROCNO

F2 - Acqui si ti on Par amet er

Dat e

20061120

Ti me

16. 22

I NSTRUM

PROBHD

PULPROG

TD

SQLVENT

NS

DS

SWH

$\mathrm{AQ}$

RG

DW

DE

TE

D1

$5 \mathrm{~mm}$ PABBO BB-
zg30
65536

$\mathrm{CDC} 3$

8278. $146 \mathrm{H}$

$0.126314 \mathrm{H}$

$3.9584243 \mathrm{~s}$

128

60.400 us 6.00 us 298. $0 \mathrm{~K}$

\section{TDO}

1. $00000000 \mathrm{se}$

$========$ CHANNEL $f$ 1 =====: NuC1

P1 $===$

10. 75 us

SFO1

$0.00 \mathrm{dt}$

SFOct 1324710 M

F2 - Processing par amet e

$\begin{array}{lr}\text { SI } & 32768 \\ \text { SF } & 400.1300211\end{array}$

WOW

SSB

LB

no

$0.00 \mathrm{HE}$

1. 00 

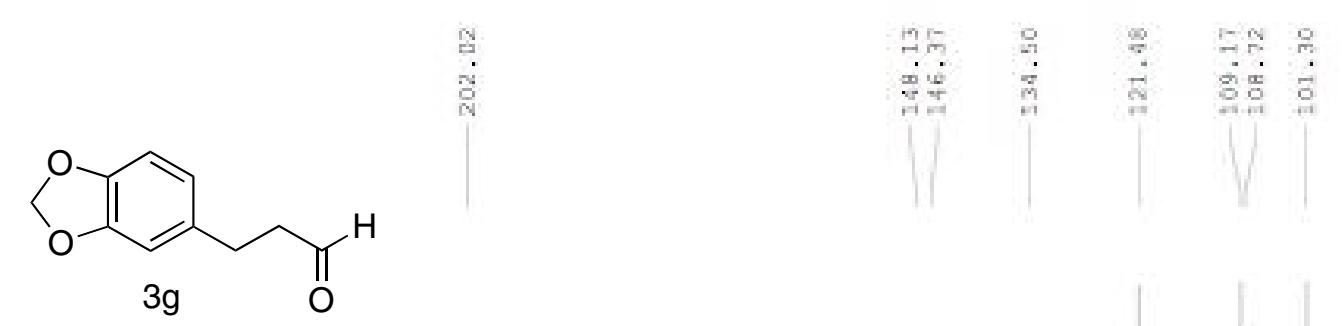

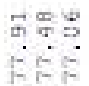

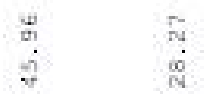

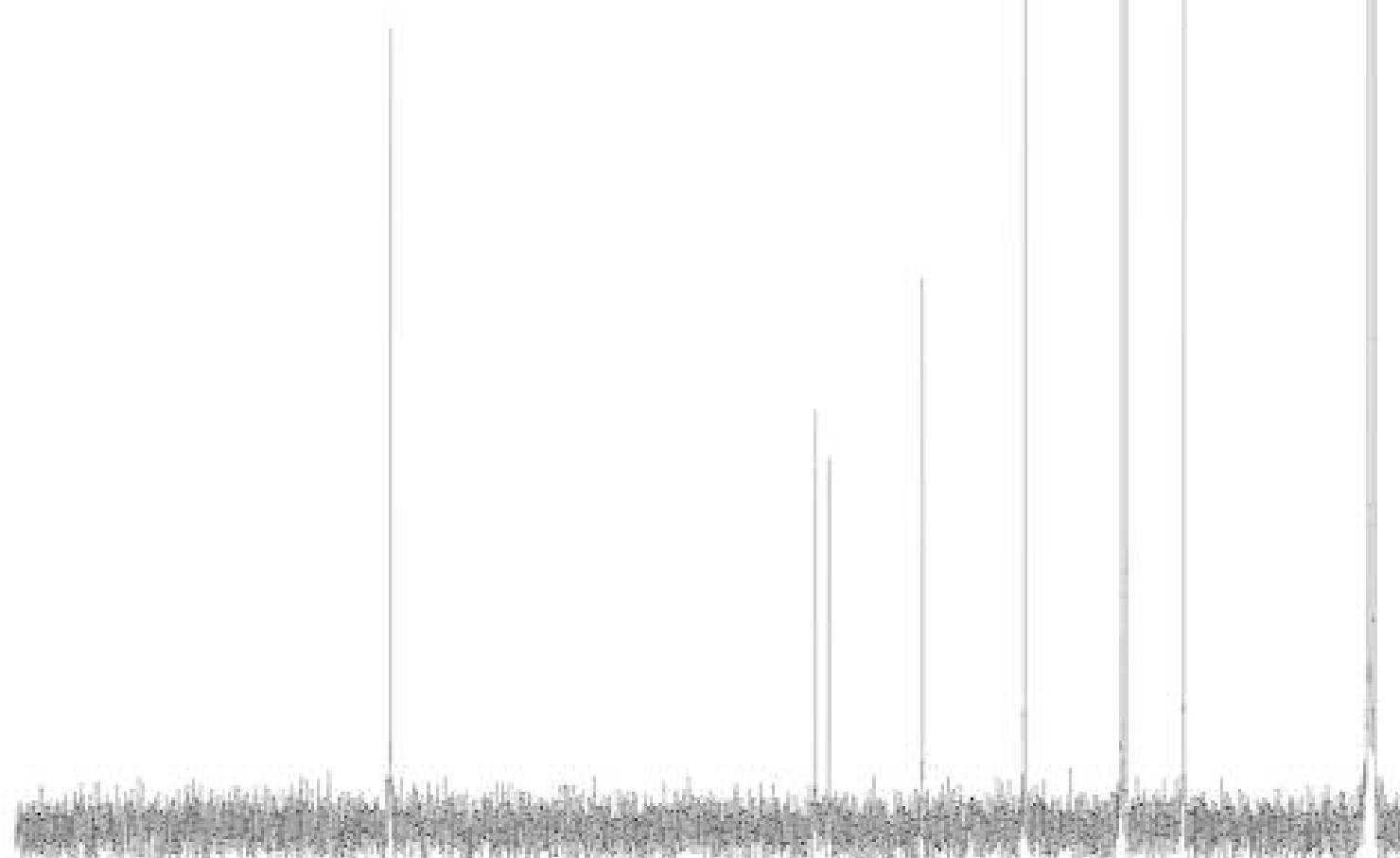

4.

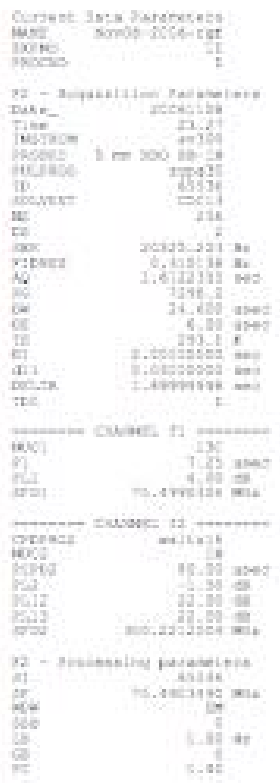

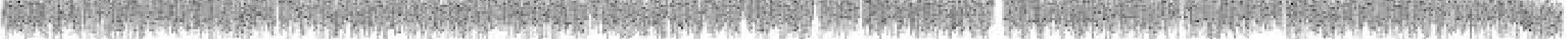

$\begin{array}{lllllllllllllllllllllllllll}240 & 290 & 220 & 210 & 200 & 190 & 280 & 170 & 160 & 150 & 140 & 130 & 120 & 110 & 100 & 90 & 80 & 70 & 60 & 59 & 40 & 30 & 20 & 10 & 0 & -10 & \text { pam }\end{array}$ 


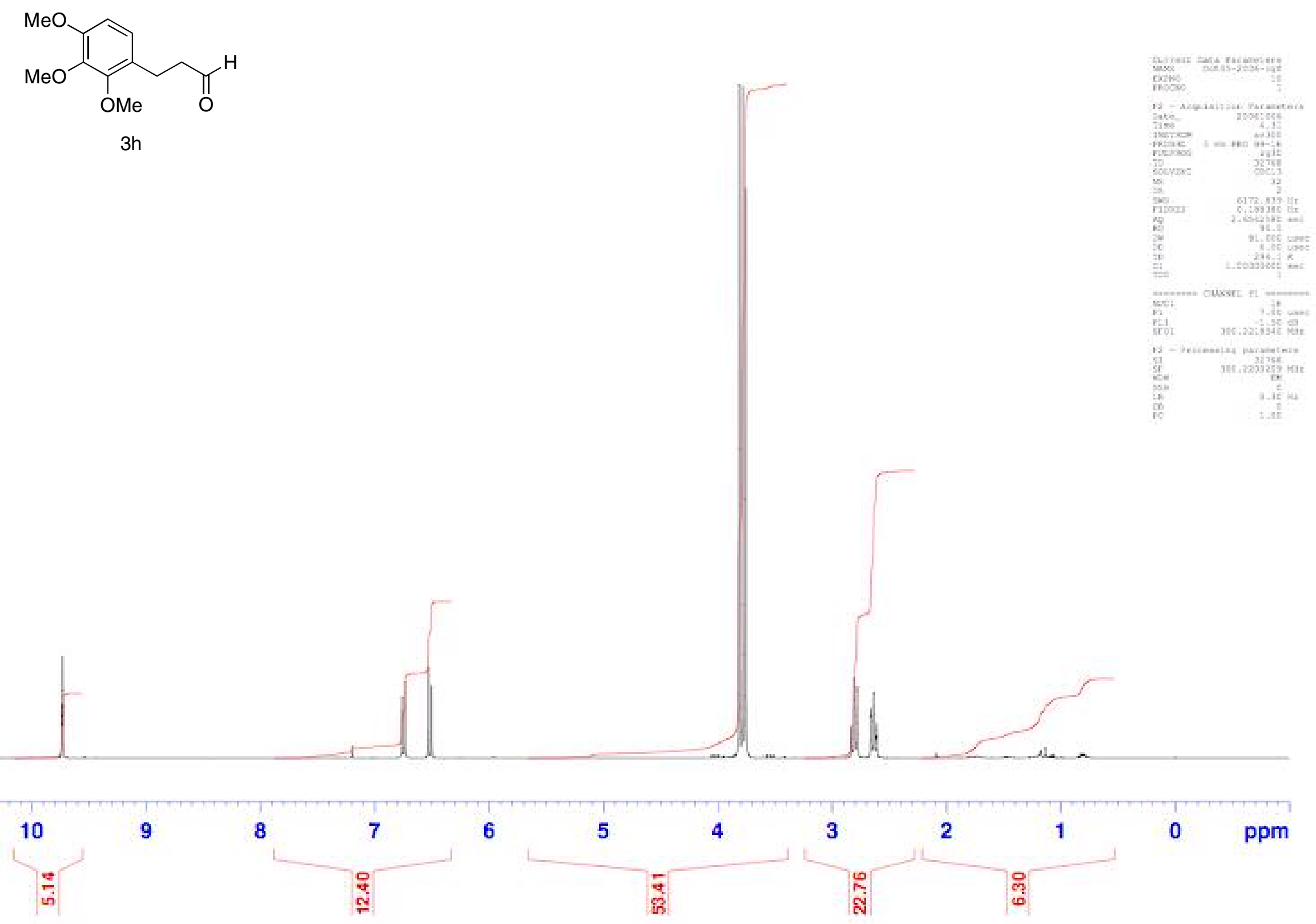




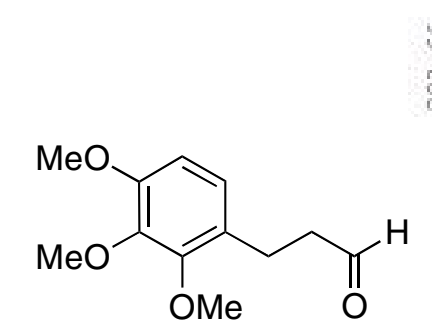

3h
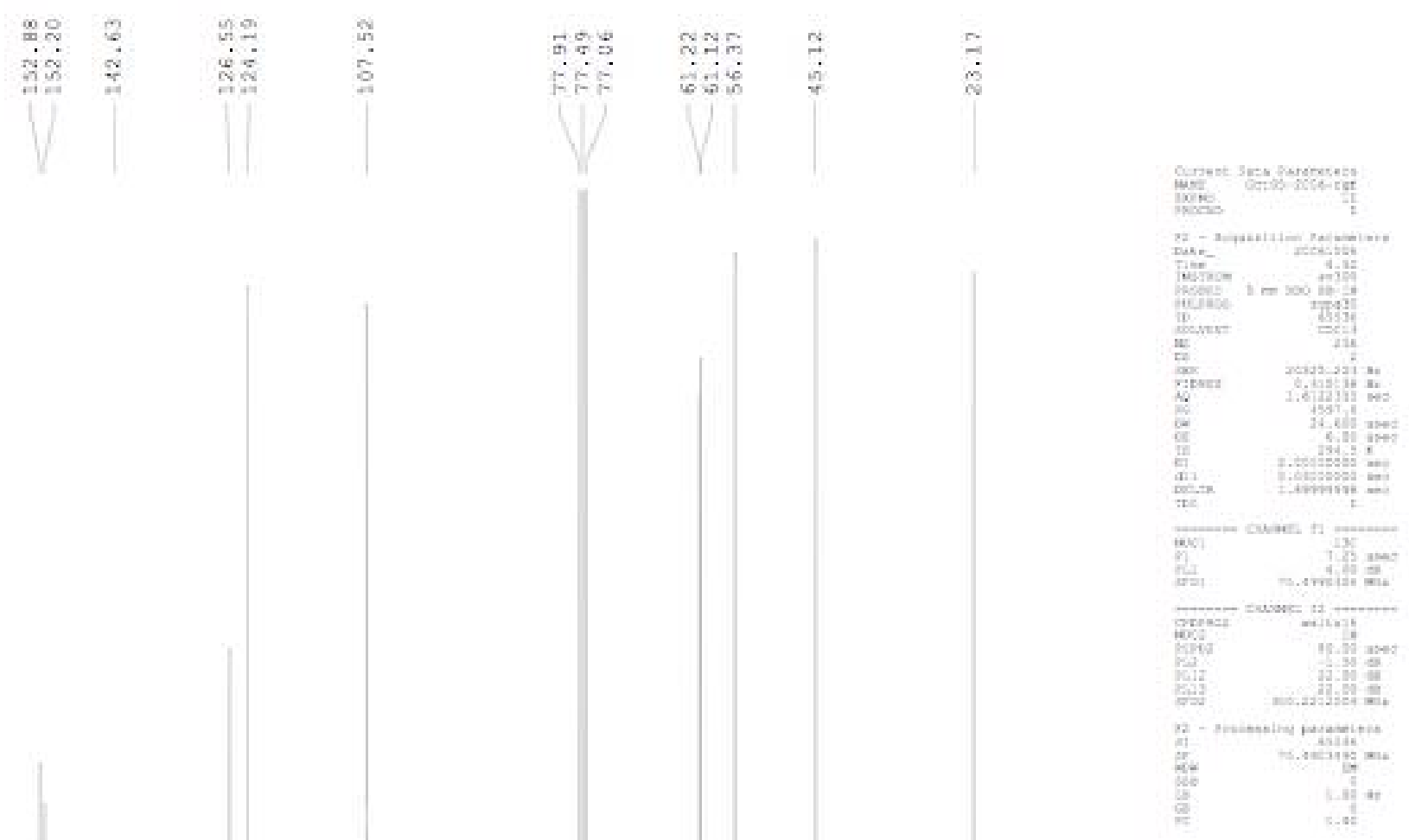

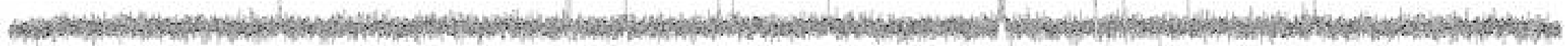

$\begin{array}{llllllllllllllllllllllllllll}240 & 290 & 220 & 210 & 200 & 190 & 280 & 170 & 160 & 150 & 140 & 130 & 120 & 110 & 100 & 90 & 80 & 70 & 60 & 50 & 40 & 30 & 20 & 10 & 0 & -10 & \text { p2m }\end{array}$ 


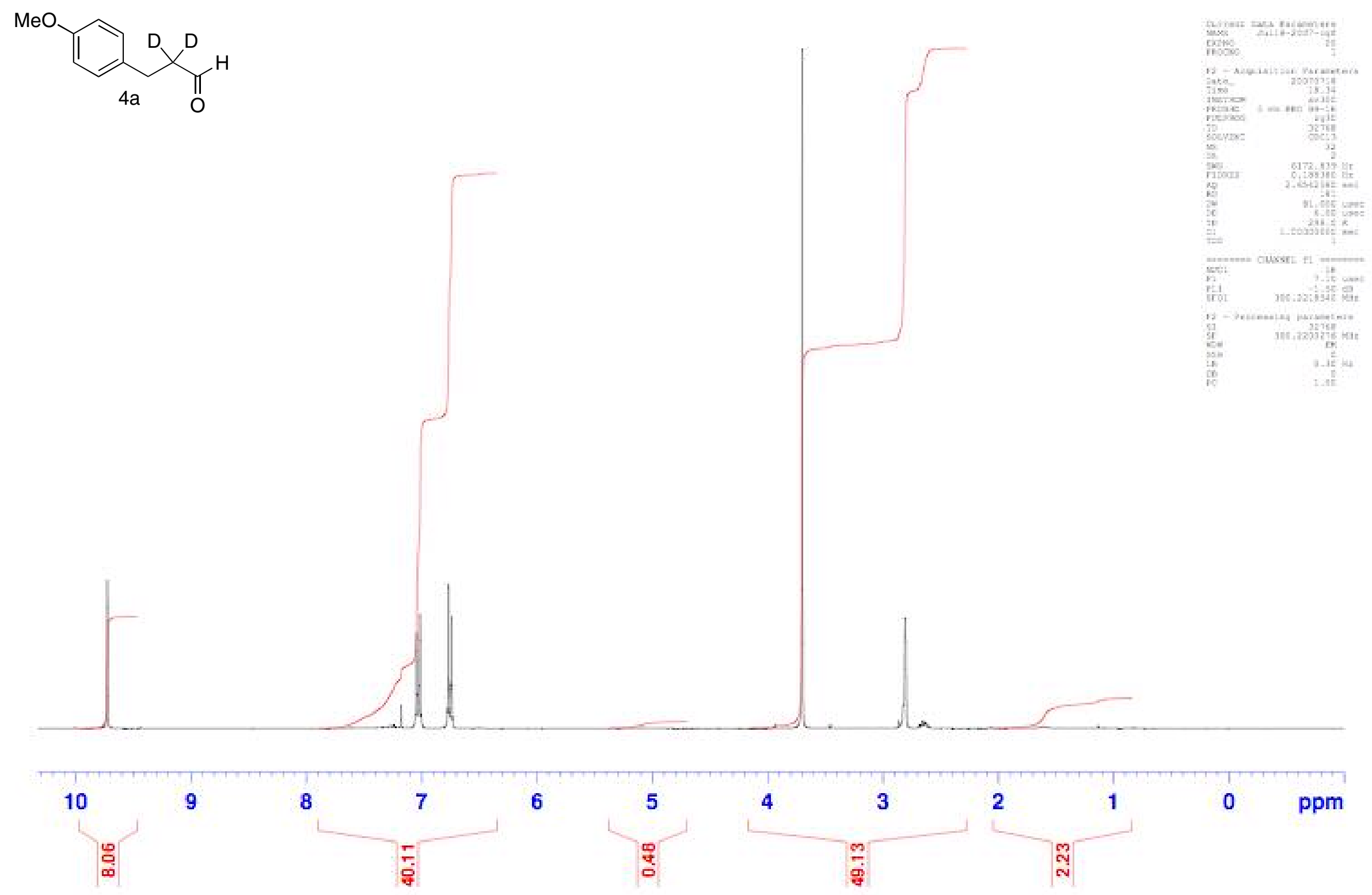


D2O

$\mathrm{MeO}$

$\underbrace{D}_{4 a} x_{0}^{D}$ $\rightarrow \underset{2}{20}$

영용

$\sqrt{ }$

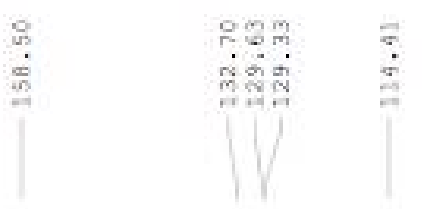

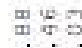

ํํำ

सित्र

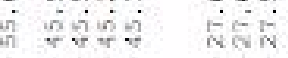

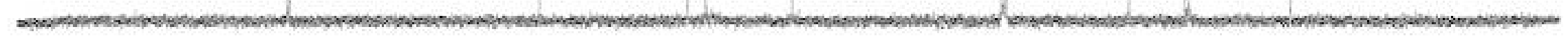

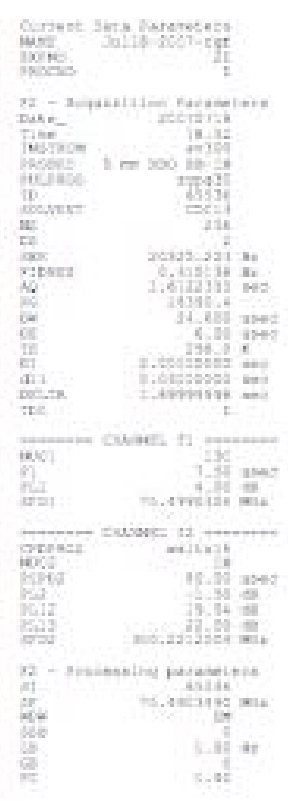

$\begin{array}{llllllllllllllllllllllllllll}240 & 290 & 220 & 210 & 200 & 190 & 280 & 170 & 160 & 150 & 140 & 130 & 120 & 110 & 100 & 90 & 80 & 70 & 60 & 59 & 40 & 30 & 20 & 10 & 0 & -10 & \text { pem }\end{array}$ 


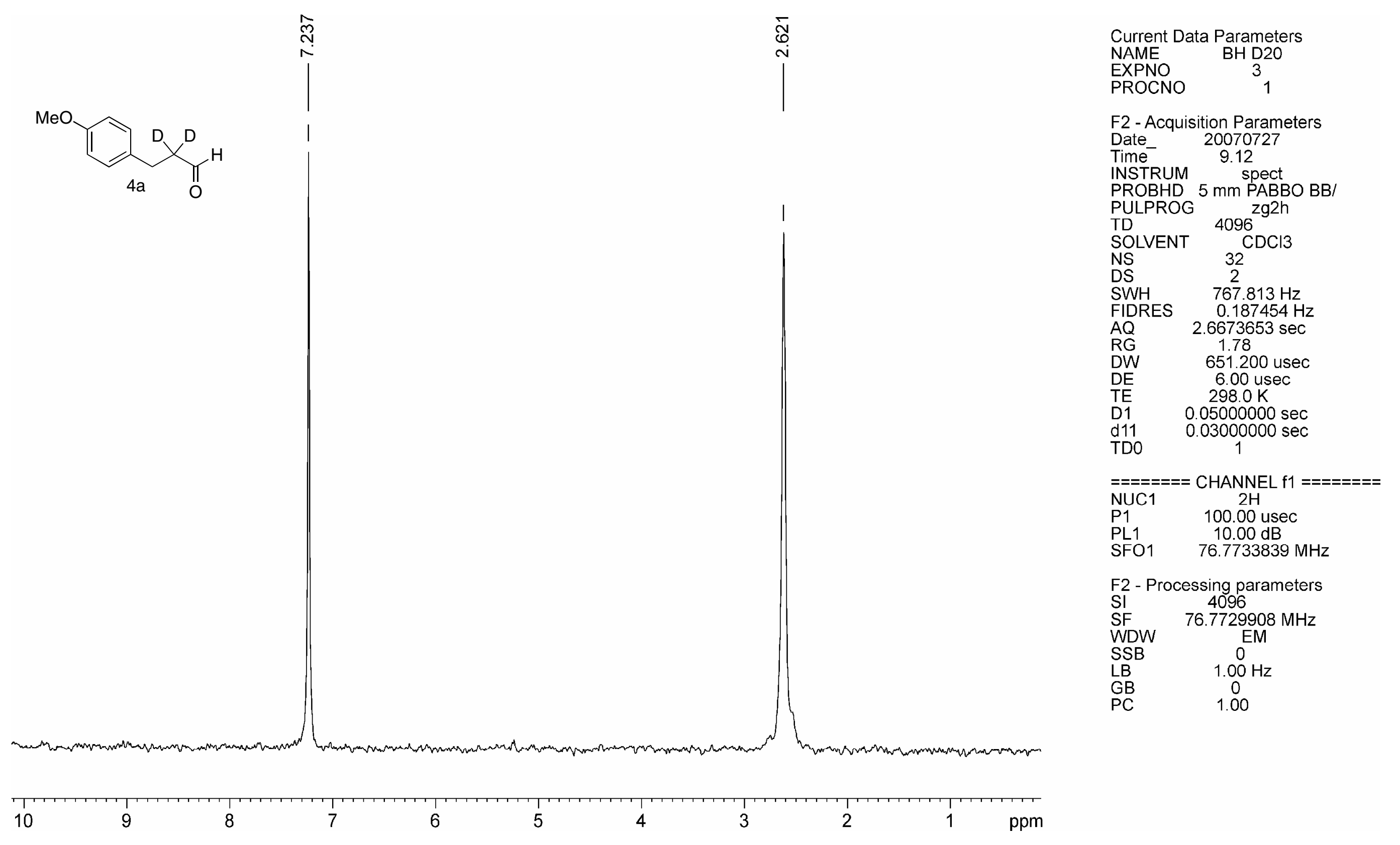




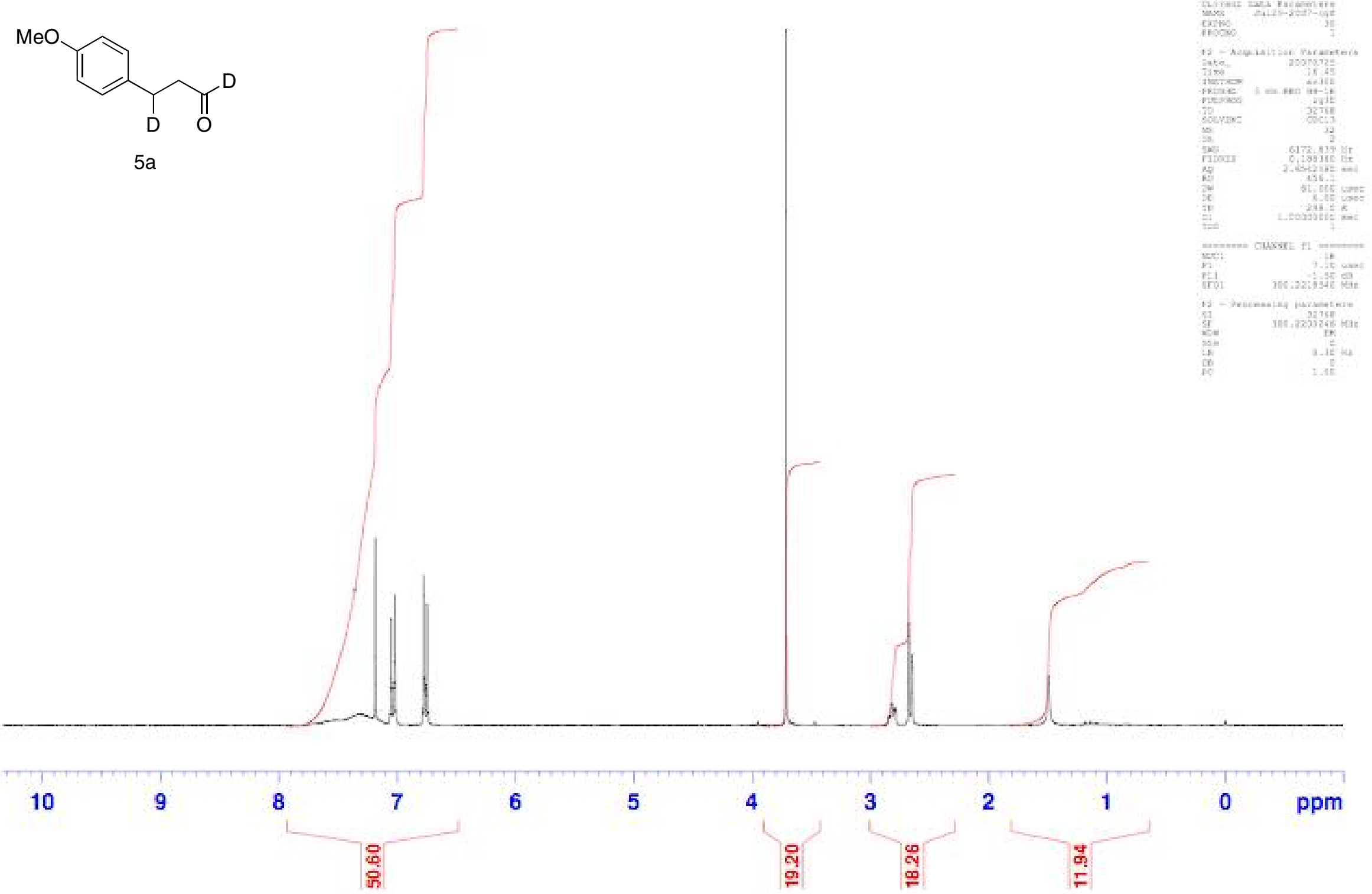


$\mathrm{MeO}$

$\overbrace{O}^{D}$

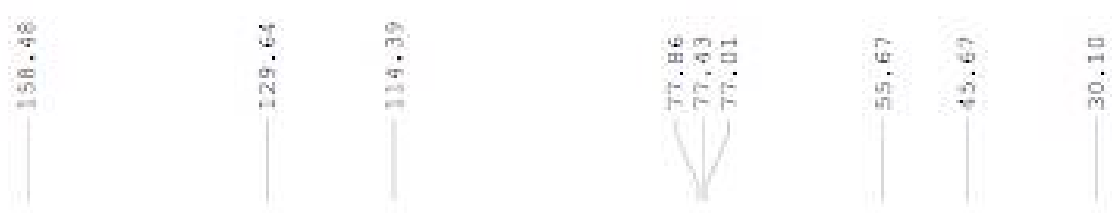

$5 a$

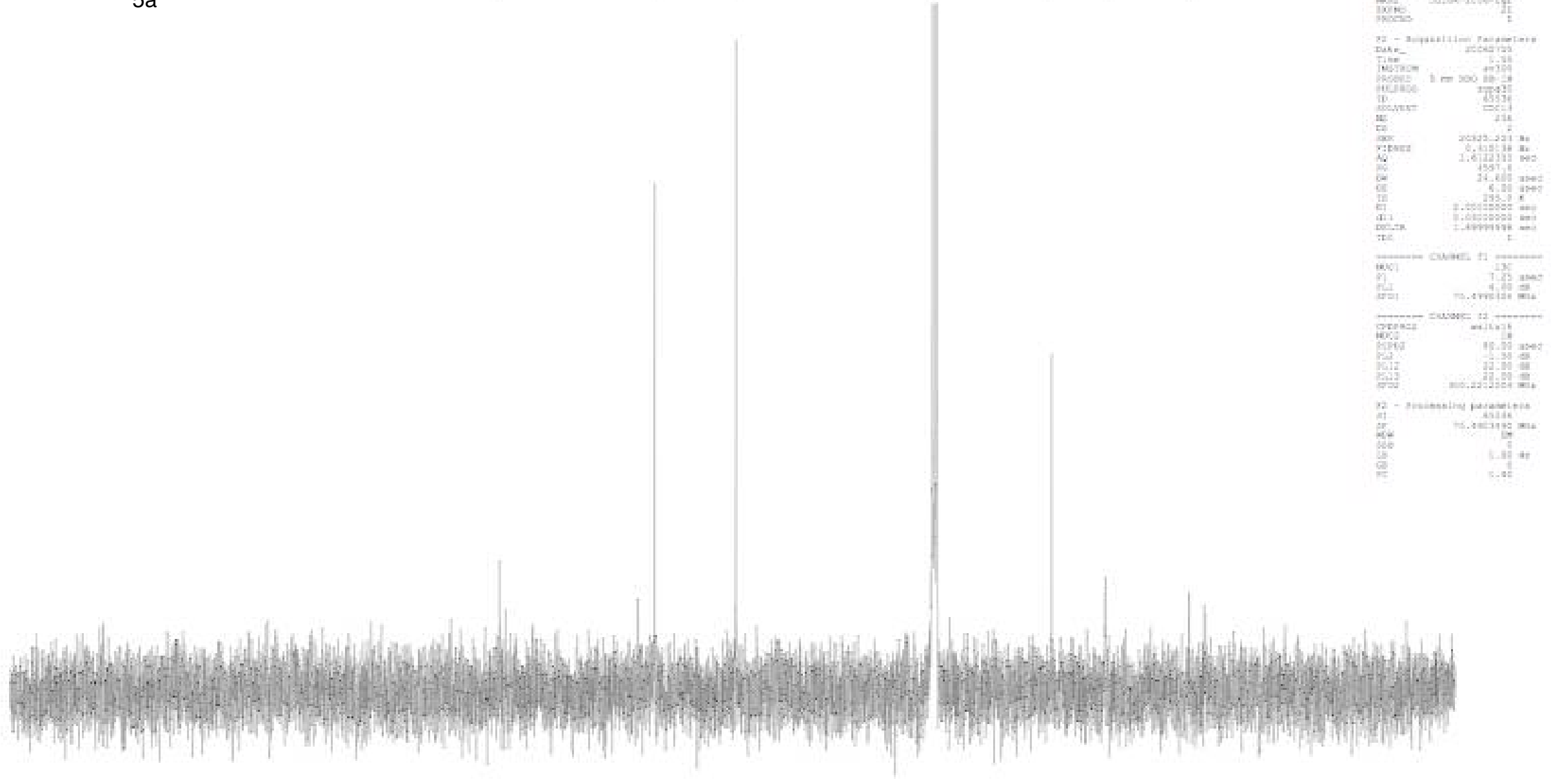

$\begin{array}{lllllllllllllllllllllllllll}240 & 290 & 220 & 210 & 200 & 190 & 280 & 170 & 160 & 150 & 140 & 130 & 120 & 110 & 100 & 90 & 80 & 70 & 60 & 59 & 40 & 30 & 20 & 10 & 0 & -10 & 59 m\end{array}$ 


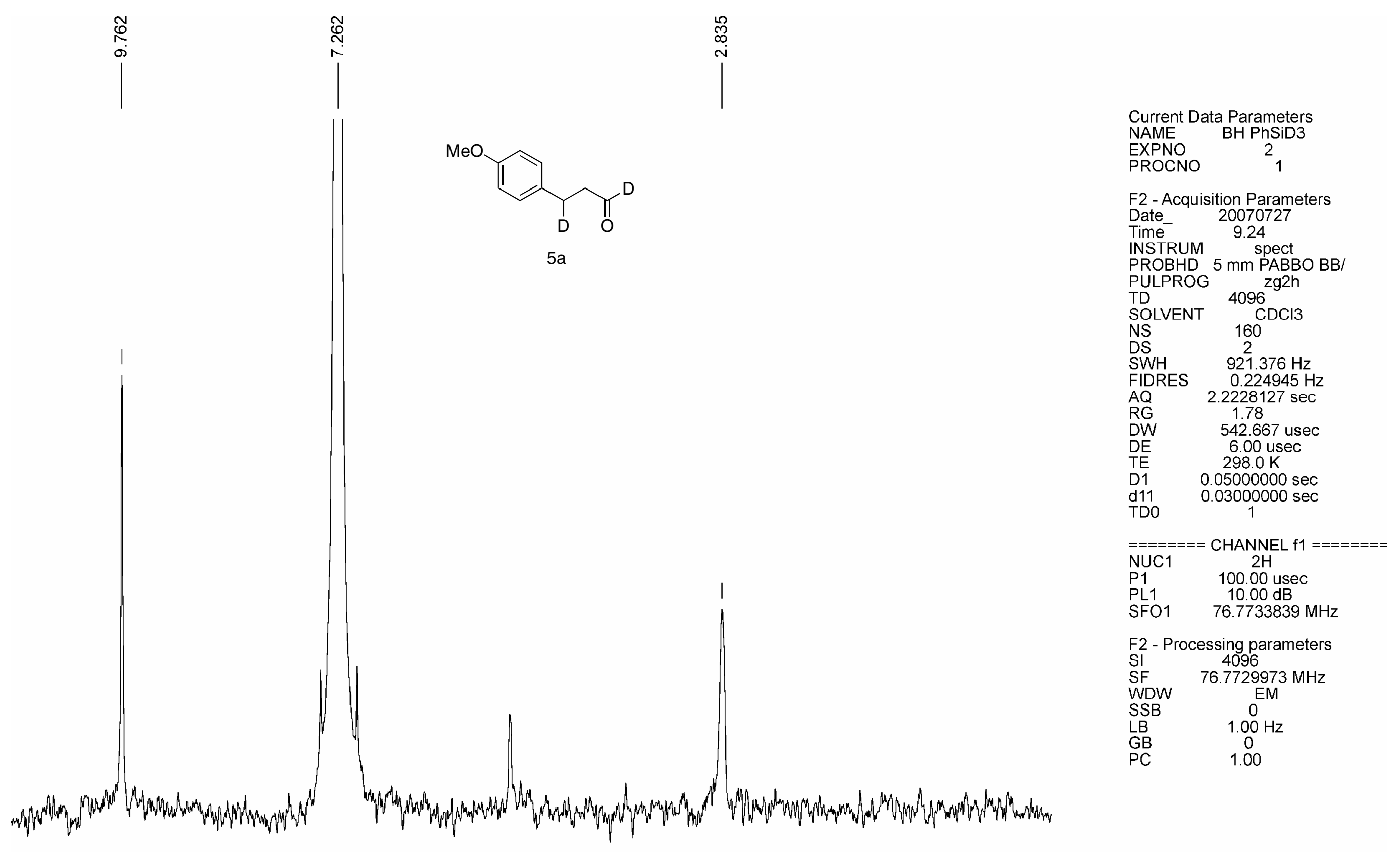


$\mathrm{MeO}$

$\underbrace{D}_{D} \int_{O}^{D}$

$6 a$

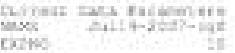

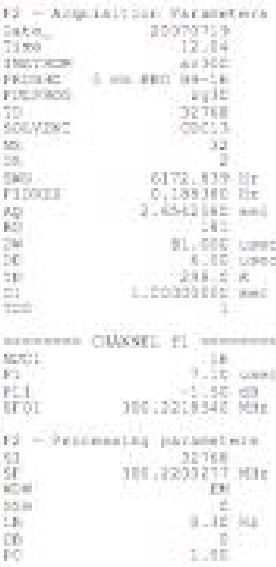

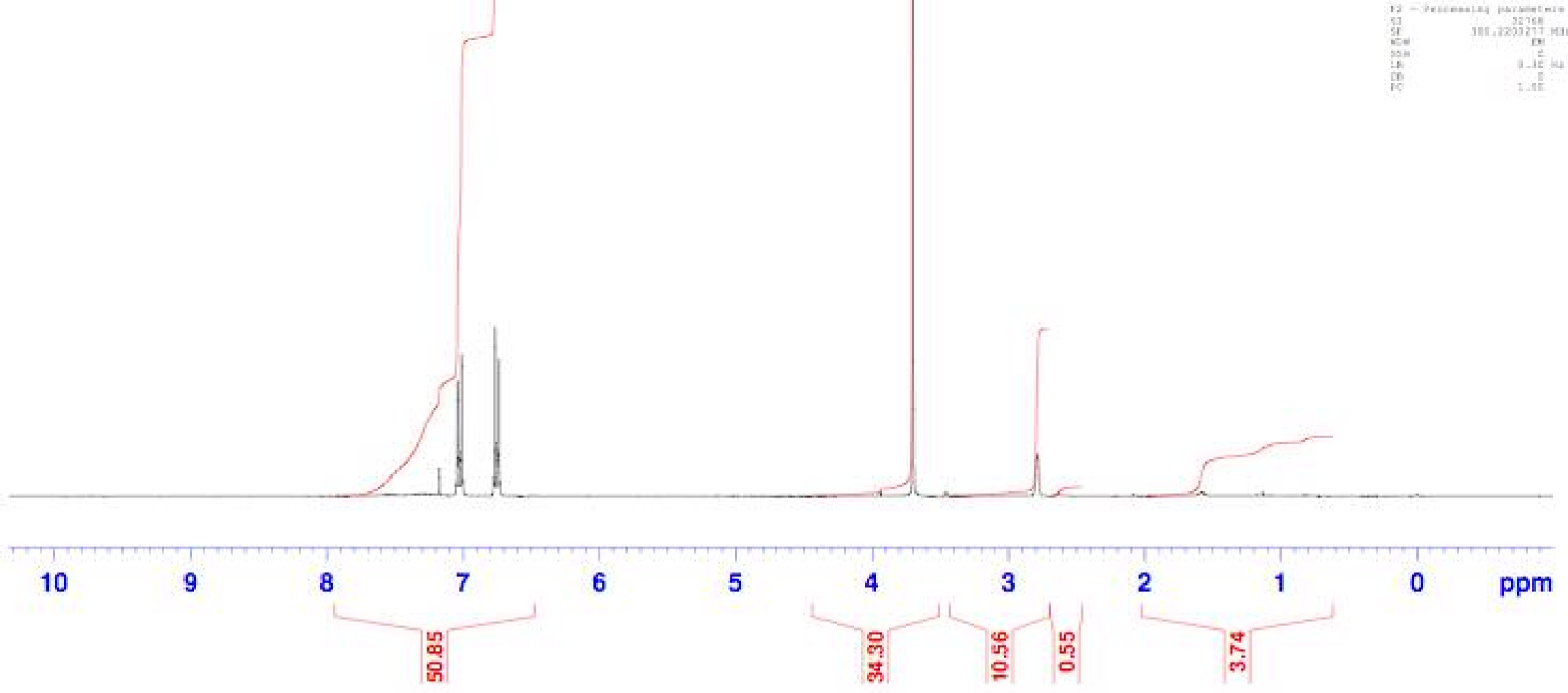




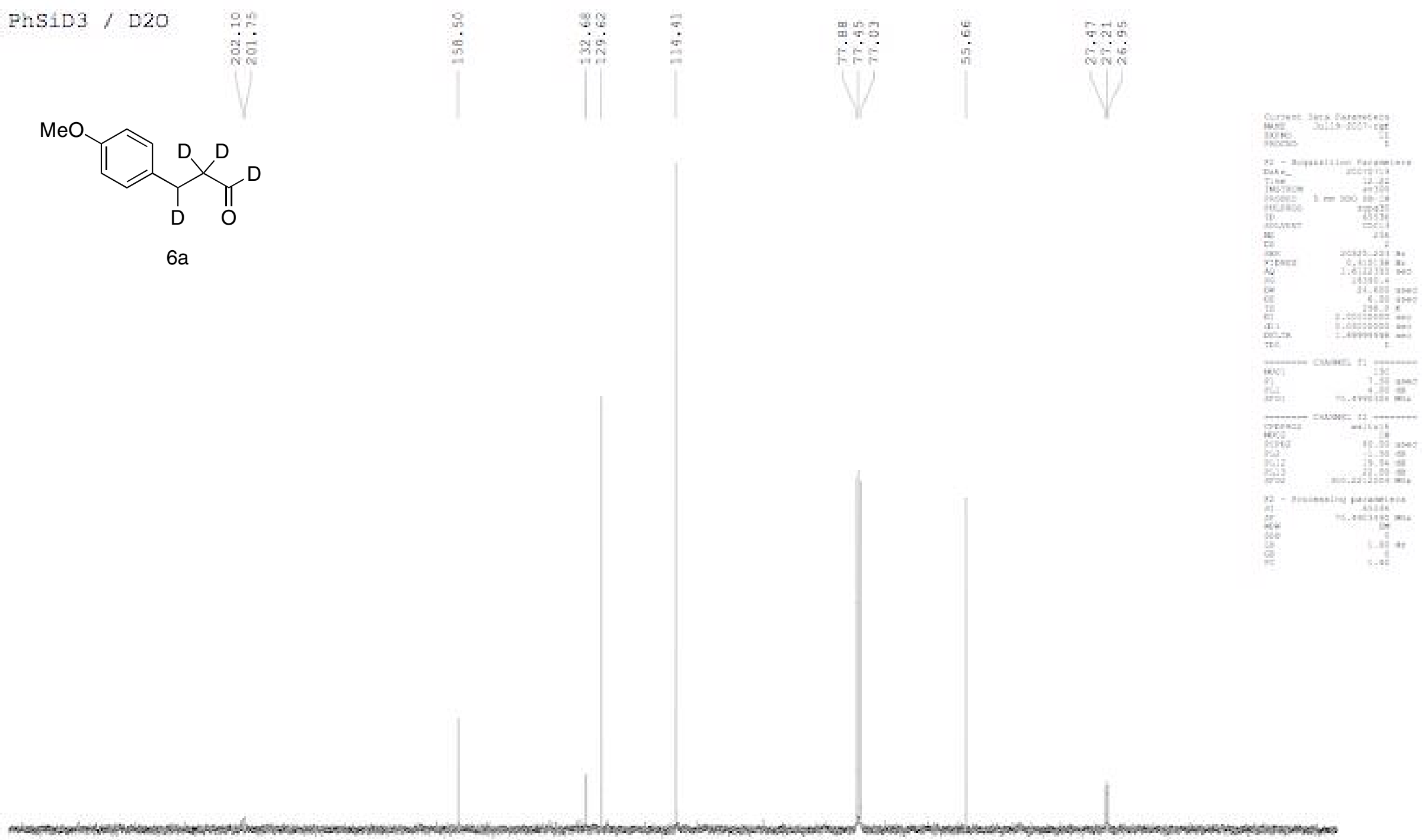

$\begin{array}{lllllllllllllllllllllllllllll}240 & 230 & 220 & 210 & 200 & 190 & 280 & 170 & 160 & 150 & 140 & 130 & 120 & 110 & 100 & 90 & 80 & 70 & 60 & 50 & 40 & 30 & 20 & 10 & 0 & -10 & \text { pam }\end{array}$ 
N N
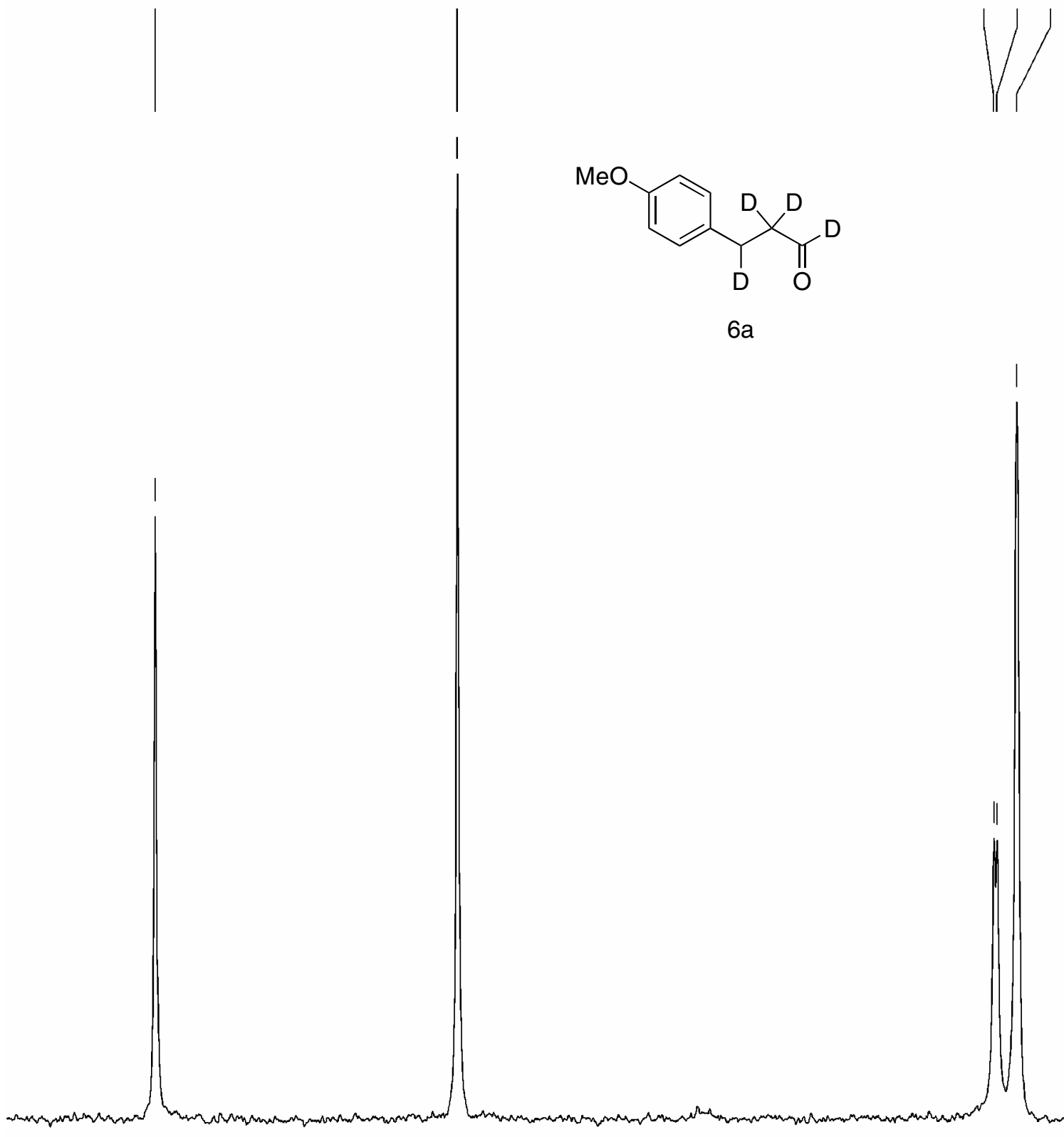

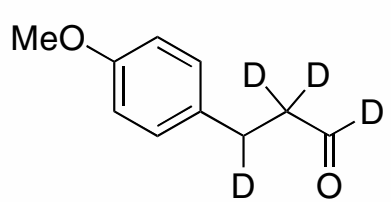

$6 a$

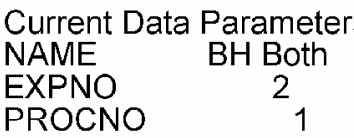

F2 - Acquisition Parameters

Date_ 20070727

Time 9.39

INSTRUM

INSTRUM 5 spect

PULPROG $\quad z g 2 h$

TD

SOLVENT

NS

DS

SWH

FIDRES

$A Q$

$\mathrm{RG}$

DW

TE

d11

dD11

$\begin{array}{lc}====== & \text { CHANNEL } f 1 \text { }======= \\ \text { NUC1 } & 2 \mathrm{H} \\ \text { P1 } & 100.00 \mathrm{usec} \\ \text { PL1 } & 10.00 \mathrm{~dB} \\ \text { SFO1 } & 76.7733839 \mathrm{MHz}\end{array}$

SFO1 $76.7733839 \mathrm{MHz}$

F2 - Processing parameters

$\begin{array}{ll}\text { SI } & 4096 \\ \text { SF } & 76.7729915 \mathrm{MHz}\end{array}$

WDW $\quad$ EM

SSB

LB

$\mathrm{GB}$

$\mathrm{PC}$

$1.00 \mathrm{~Hz}$

0
1.00 
1. F. Bigi, S. Carloni, L. Ferrari, R. Maggi, A. Mazzacani and G. Sartori, Tetrahedron Lett., 2001, 42, 5203-5205.

2. B. Hin, P. Majer and T. Tsukamoto, J. Org. Chem., 2002, 67, 7365-7368.

3. E. Fillion, A. M. Dumas and S. A. Hogg, J. Org. Chem., 2006, 71, 9899-9902.

4. N. B. Darvatkar, A. R. Deorukhkar, S. V. Bhilare and M. M. Salunkhe, Syn.Commun., 2006, 36, 3043-3051.

5. K. Tanaka, X. Chen, T. Kimura and F. Yoneda, Chem. \& Pharm. Bull., 1988, 36, 6069.

6. V. Armstrong, O. Soto, J. A. Valderrama and R. Tapia, Syn. Commun., 1988, 18, $717-$ 725 .

7. D. Q. Shi, Y. C. Wang, Z. S. Lu and G. Y. Dai, Syn. Commun., 2000, 30, 713-726.

8. F. Rouessac and A. Leclerc, Syn. Commun., 1993, 23, 2709-2715.

9. M. Kimura and M. Seki, Tetrahedron Lett., 2004, 45, 3219-3223.

10. R. L. Blankespoor, T. DeVries, E. Hansen, J. M. Kallemeyn, A. M. Klooster, J. A. Mulder, R. P. Smart and D. A. V. Griend, J. Org. Chem., 2002, 67, 2677-2681.

11. R. Schobert, S. Siegfried and G. J. Gordon, J. Chem. Soc.-Perkin Trans. 1, 2001, 2393-2397. 